\title{
The Branch Cut and Quasi-normal Modes at large imaginary frequency in Schwarzschild space-time
}

\author{
Marc Casals* \\ Perimeter Institute for Theoretical Physics, Waterloo, Ontario, Canada N2L $2 Y 5$ \\ Department of Physics, University of Guelph, Guelph, Ontario, Canada N1G 2W1 and \\ School of Mathematical Sciences and Complex $\&$ Adaptive Systems Laboratory, \\ University College Dublin, Belfield, Dublin 4, Ireland \\ Adrian Ottewill \\ School of Mathematical Sciences and Complex $\&$ Adaptive Systems Laboratory, \\ University College Dublin, Belfield, Dublin 4, Ireland
}

(Dated: March 15, 2021)

\begin{abstract}
The 'retarded' Green function for fields propagating on a Schwarzschild black hole spacetime possesses a branch cut on the complex frequency plane. Classically, the branch cut is important, for example, in order to fully determine the response of the black hole to a linear field perturbation. The branch cut is also useful for the calculation of the self-force on a point particle moving in the Schwarzschild background. In this paper we use techniques of analytic-continuation to the complex plane of the radial coordinate in order to calculate the branch cut contribution to the Green function in the limit of large imaginary frequency. It is expected that the contribution of this frequency regime to the perturbation response and to the self-force will be mostly for short time intervals. We also determine the highly-damped quasinormal mode frequencies for electromagnetic perturbations in Schwarzschild for the first time (previously only the leading imaginary part was known), which seem to have a 'deep connection' with the branch cut. We find that these frequencies behave like $\omega_{l n}=-\frac{i n}{2}-\frac{i[\ell(\ell+1)]^{2}}{2 n}+\frac{\pi^{1 / 2}(1-i)[\ell(\ell+1)]^{3}}{2^{3 / 2} n^{3 / 2}}+O\left(n^{-2}\right)$. The highly-damped quasinormal modes are particularly interesting for theories of quantum gravity in that they are believed to probe the small scale structure of the spacetime.
\end{abstract}

\section{INTRODUCTION}

In the investigation of spin-field perturbations on a curved spacetime, the 'retarded' Green function plays a crucial rôle. For example, the full evolution in time of some initial data may be determined by integrating over space the 'retarded' Green function convolved with the initial data. Also, the self-force acting on a point particle moving on a background spacetime may be calculated via an integration of the 'retarded' Green function over the whole past worldline of the particle [1. Similarly in quantum field theory the covariant commutation relations are determined by the 'advanced' and 'retarded' Green functions. While in some contexts such as renormalization a knowledge of the short-distance behaviour of the Green function is sufficient, in other contexts the knowledge of the global behaviour of the Green function is important.

The 'retarded' Green function for linear field perturbations of the Schwarzschild black hole spacetime can be expressed as a multipole decomposition together with a Fourier integral over the frequencies just above the real axis. In the impressive work of [2, Leaver deforms the frequency integral into a contour in the complex-frequency plane and he investigates three distinct contributions to the Green function coming from: (1) a high-frequency arc, (2) the poles (quasinormal modes) in the lower plane, and (3) a branch cut (BC) along the negative imaginary axis (NIA). Under a linear field perturbation, the high-frequency arc leads to a prompt response; the poles lead to the well-known quasinormal mode 'ringing' (this 'ringing' was observed for the first time in [3]); the BC leads to, at least, a power-law tail decay with time [4, 5. While the quasinormal modes (QNMs) have been extensively investigated, most work on the BC has been limited to small frequency on the NIA (and usually also large radial coordinates), which is precisely the regime that gives the power-law tail - see, e.g. 2, 6. The $\mathrm{BC}$, however, is also expected 2 to have a significant contribution at 'early' times, just after the start of the prompt response from the high-frequency

*Electronic address: mcasals@perimeterinstitute.ca; mcasals@uoguelph.ca; marc.casals@ucd.ie

$\dagger$ Electronic address: adrian.ottewill@ucd.ie 
arc. In fact, at 'very early' times, i.e., before the prompt response, the separate contributions from the high-frequency arc, QNMs and BC might all separately diverge with their divergences cancelling each other out so that the complexfrequency contour integration is still valid. It is expected that these BC contributions at 'early' and 'very early' times come from the mid- and high-frequency regimes of the BC on the NIA, which might also bring about other yet-unsuspected contributions. To the best of our knowledge, these mid- and high-frequency regimes of the BC have only been investigated, respectively, in [7, 9] and in Maassen van den Brink's [10], and it was done solely for the case of gravitational perturbations. In [10], Maassen van den Brink investigated the BC in Schwarzschild for high-frequencies using a method based on an analytic continuation to the complex plane of the radial coordinate $r$. This method is mirrored on the method used in [11, 12] for the calculation of highly-damped QNMs, i.e., QNMs for large overtone index $n$, which therefore lie far down in the lower frequency plane.

On the quantum side, highly-damped QNMs have attracted considerable attention since attempts [13 15] have been made at linking them to the area quantization of a black hole [16, 17. For example, in [18, the imaginary part of the highly-damped QNMs is shown to be related to the exponential redshift of the wave modes close to the horizon. In [19], highly-damped QNMs in Kerr spacetime have been interpreted as semiclassical bound states along a specific contour in the complex-r plane; they speculate that QNMs and another set of modes (the so-called totally-transmitted modes) correspond to different sets of microscopic degrees of freedom which, when they interact, produce Hawking radiation. Separately, in 20] they have shown that highly-damped QNMs probe the short length scale structure of the Schwarzschild black hole spacetime by calculating them in the context of a 'quantum-corrected' black hole.

To set the current work in context we here give a succinct review of the -mostly- analytic results obtained in the literature for highly-damped QNM frequencies in the Schwarzschild spacetime for various spin- $s$ fields. Motl and Neitzke [11, 12 originally calculated analytically the leading-order of the highly-damped QNMs for $s=0$ and 2 ([12] also predicted the dependence of their next-to-leading order term on the 'angular momentum number' $\ell$ ). Subsequently, Maassen van den Brink [10] for $s=2$ and Musiri and Siopsis [21] for $s=0$ and 2 confirmed the leading-order behaviour and obtained the next-to-leading order highly-damped QNMs. In [21, 22] they also give the leading-order for the imaginary part -though not for the real part- of the QNM frequencies for $s=1 / 2,1$ and $3 / 2$. Finally, in 23 they obtain the leading-order and next-to-leading order QNMs for $s=1 / 2$ and $s=5 / 2$. To the best of our knowledge, the leading-order for the real part of the QNM frequencies for $s=1$ and $s=3 / 2$ remains unknown, and only the leading order of their imaginary parts is known. The case of electromagnetic $(s=1)$ perturbations is particularly interesting since the real part of the spin-1 highly-damped QNM frequencies have been expected to approach the NIA, thus hinting at an unexplored possible connection between the highly-damped QNMs and the BC. Indeed, in [21, 23. they show that the real part of the electromagnetic QNM frequencies can only go at most like $n^{-1}$ for $n \rightarrow \infty$, and in 24 they find numerical indications that it goes like $n^{-3 / 2}$, with a coefficient which is a $3 r d$ order polynomial in $\ell(\ell+1)$ (with undetermined polynomial coefficients). We will confirm this behaviour and determine the polynomial coefficients in Sec.IV. This behaviour is in contrast with the $O(1)$ behaviour of the corresponding QNM frequencies for $s=0$ and 2 and faster-decaying than the $O\left(n^{-1 / 2}\right)$ for $s=1 / 2$ and $5 / 2$. See 25] for a thorough and recent review of QNMs in different spacetimes; see also [26].

As mentioned, the contributions of the mid- and high-frequency regimes of the BC still remain largely unexplored. In the present paper we use the method in [10] (which was restricted to $s=2$ ) to carry out an asymptotic calculation in the high-frequency regime of the BC contribution to the 'retarded' Green function in Schwarzschild for fields of spin $s=0$ (scalar) and 1 (electromagnetic). We also calculate the highly-damped QNM frequencies for electromagnetic perturbations for the first time in the literature (other than the leading-order of the imaginary part, which is known): we calculate these frequencies up to order $n^{-2}$, that is, leading-order for the real part and up to two orders after leading-order for the imaginary part. The feature that these QNMs approach the NIA 'so fast' has made them very unyielding: like we show in this paper, in order to obtain the leading-order behaviour for large-frequency for spin-1 we are required to go up to two higher orders in perturbation theory than we are required for spins 0 and 2. Electromagnetic QNMs are increasingly important, since the detection of the electromagnetic counterpart of the gravitational wave emission by astrophysical sources might play a valuable rôle for localizing the source and obtaining further information about it [27]. For completeness, we also reproduce in this paper the results in [10] for the case of $s=2$ (gravitational) and in [21] for the corresponding spin-0 QNM frequencies.

One particular physical application of the BC contribution to the 'retarded' Green function is the calculation of the corresponding contribution to the response of the Schwarzschild black hole to some initial perturbation. In this paper we investigate the rôle played by the high-frequency part of both the BC and the QNM contributions to such a response in the case of a spin- $s(=0,1,2)$ field sourced by: (1) initial data of compact support and (2) a non-compact Gaussian distribution in the 'tortoise' radial coordinate.

In two other papers [28, 29], we will investigate, using completely different methods, the BC in Schwarzschild in 
the mid-frequency and the small-frequency regimes along the NIA. In most of the figures in the present paper we plot the various quantities required for the calculation of the $\mathrm{BC}$ contribution to the Green function. In these figures we compare the high-frequency asymptotics obtained here with the results using the independent methods which we will present in [29]. The method in [29] has good convergence in a 'mid'-frequency regime which overlaps with the 'high'-frequency regime, as shown here; it also overlaps with the 'small'-frequency regime, as we will show in [28].

This paper is organized as follows: In Sec III we give the general formulae for the BC contribution to the 'retarded' Green function. In Sec III we perform the asymptotic analysis of the BC Green function for high-frequency along the NIA. In Sec $[\mathrm{IV}$ we calculate the highly-damped QNMs. In Sec $\mathrm{V}$ we investigate the contribution from the highfrequency regime of both the $\mathrm{BC}$ and the QNMs to the black hole response to an initial perturbation. In Sec VI we present some conclusions.

\section{BRANCH CUT CONTRIBUTION TO THE GREEN FUNCTION}

The 'retarded' Green function for linear field perturbations in the Schwarzschild spacetime can be expressed in terms of a multipole decomposition together with a Fourier transform as

$$
\begin{aligned}
& G_{r e t}\left(x, x^{\prime}\right)=\sum_{\ell=0}^{\infty}(2 \ell+1) P_{\ell}(\cos \gamma) G_{\ell}^{r e t}\left(r, r^{\prime} ; \Delta t\right), \quad G_{\ell}^{r e t}\left(r, r^{\prime} ; \Delta t\right) \equiv \frac{1}{2 \pi} \int_{-\infty+i c}^{\infty+i c} d \omega G_{\ell}\left(r, r^{\prime} ; \omega\right) e^{-i \omega \Delta t}, \quad \Delta t \equiv t-t^{\prime}, \\
& G_{\ell}\left(r, r^{\prime} ; \omega\right)=\frac{f_{\ell}\left(r_{<}, \omega\right) g_{\ell}\left(r_{>}, \omega\right)}{W(\omega)}, \quad r_{>} \equiv \max \left(r, r^{\prime}\right), r_{<} \equiv \min \left(r, r^{\prime}\right) \\
& W(\omega) \equiv W\left[g_{\ell}(r, \omega), f_{\ell}(r, \omega)\right]=g_{\ell}(r, \omega) \frac{d f_{\ell}(r, \omega)}{d r_{*}}-f_{\ell}(r, \omega) \frac{d g_{\ell}(r, \omega)}{d r_{*}}
\end{aligned}
$$

where $c>0, t$ and $r$ are - respectively - the time and radial Schwarzschild coordinates, $r_{*}=r+r_{h} \ln (\bar{r}-1)$ is the so-called 'tortoise' radial coordinate, $r_{h}=2 M$ is the radius of the event horizon, $M$ is the mass of the black hole and $\gamma$ is the angle between the spacetime points $x$ and $x^{\prime}$. Note that the physical region $r \in\left(r_{h}, \infty\right)$ corresponds to $r_{*} \in(-\infty, \infty)$. A bar over a quantity indicates that the quantity has been made dimensionless via an appropriate factor of $r_{h}$, e.g., $\bar{r} \equiv r / r_{h}, \bar{\omega} \equiv \omega r_{h}, \bar{t} \equiv t / r_{h}$, etc. The function $W(\omega)$ is the Wronskian of the two linearly independent solutions $f_{\ell}$ and $g_{\ell}$ of the following homogeneous radial ODE:

$$
\begin{aligned}
& {\left[\frac{d^{2}}{d r_{*}^{2}}+\omega^{2}-V(r)\right] \psi_{\ell}(r, \omega)=0} \\
& V(r) \equiv\left(1-\frac{r_{h}}{r}\right)\left[\frac{\lambda}{r^{2}}+\frac{r_{h}\left(1-s^{2}\right)}{r^{3}}\right], \quad \lambda \equiv \ell(\ell+1)
\end{aligned}
$$

The parameter $s$ denotes the spin of the field: $s=2$ corresponds to axial - also called 'odd' - gravitational perturbations (in which case Eq. (2.2) becomes the Regge-Wheeler equation [30]), $s=1$ to electromagnetic perturbations [31] and $s=0$ to scalar perturbations 4 , 5. The ODE Eq. 2.2. has two regular singular points at $r=0, r_{h}$ and an irregular singular point at $r=\infty$. These singularities in the ODE generally cause the radial solutions to have branch points at $r=0$ and $r_{h}$ in the complex- $r$ plane. For real $\omega$, the solutions $f_{\ell}$ and $g_{\ell}$ are uniquely determined by the boundary conditions:

$$
\begin{array}{ll}
f_{\ell}(r, \omega) \sim e^{-i \omega r_{*}}, & r_{*} \rightarrow-\infty, \\
g_{\ell}(r, \omega) \sim e^{+i \omega r_{*}}, & r_{*} \rightarrow+\infty .
\end{array}
$$

Strictly speaking, the limits in Eq. 2.3 should actually be $\bar{r}_{*} \rightarrow \mp \infty$, but following standard conventions we denote them by $r_{*} \rightarrow \mp \infty$, with a certain abuse of language. We also have

$$
f_{\ell}(r, \omega) \sim A_{\ell, \omega}^{\text {out }} e^{+i \omega r_{*}}+A_{\ell, \omega}^{\text {in }} e^{-i \omega r_{*}}, \quad r_{*} \rightarrow+\infty,
$$

where $A_{\ell, \omega}^{\text {out }} \in \mathbb{C}$ and $A_{\ell, \omega}^{\text {in }} \in \mathbb{C}$ are reflection and incidence coefficients, respectively. It is immediate that the Wronskian is equal to $W(\omega)=-2 i \omega A_{\ell, \omega}^{i n}$. The boundary condition 2.3 also define $f_{\ell}$ and $g_{\ell}$ unambiguously for $\operatorname{Im}(\omega) \geq 0$ when $r_{*} \in \mathbb{R}$; these solutions are then defined for $\operatorname{Im}(\omega)<0$ by analytic continuation (see [9] for details on the region of 
validity of these boundary conditions including $r_{*}$ as well as $\left.\omega\right)$. On the NIA, the boundary condition $(2.3)$ becomes meaningless since it does not allow one to exclude the exponentially-decaying solution, $e^{+i \omega r_{*}}$ for $f_{\ell}$ and $e^{-\imath \omega r_{*}}$ for $g_{\ell}$. We are therefore motivated to analytically continue to the complex- $r$ plane, and impose these boundary conditions in the regions $\operatorname{Re}\left(-i \omega r_{*}\right) \leq 0$ for $f_{\ell}$ and $\operatorname{Re}\left(i \omega r_{*}\right) \leq 0$ for $g_{\ell}$, where they define the solutions uniquely (see also [32, 33]).

Leaver 34] has shown that $g_{\ell}(r, \omega)$ has a branch cut (BC) which can be naturally taken to run along the line $\omega r: 0 \rightarrow-\infty \cdot i$. If $r>0$, then $g_{\ell}(r, \omega)$ has a branch point at $\omega=0$ and a BC along the NIA, $\omega: 0 \rightarrow-\infty \cdot i$. In [35, 36] they have shown that the existence of a BC in the complex- $\omega$ plane is linked to the asymptotic behaviour of the radial potential: the exponentially-decaying Schwarzschild potential $V(r) \sim e^{\bar{r}_{*}-1}\left[\lambda+1-s^{2}\right] / r_{h}^{2}$ as $r_{*} \rightarrow-\infty$ leads to poles in $f_{\ell}$ on the NIA (these poles, however, are cancelled out in $G_{\ell}$ by the corresponding poles in $W(\omega)$ ), whereas its slower-than-exponential decay (with the exception of the centrifugal barrier) $V(r)-\lambda / r_{*}^{2} \sim 2 \lambda r_{h} \ln \left(\bar{r}_{*}\right) / r_{*}^{3}$ as $r_{*} \rightarrow \infty$ leads to the $\mathrm{BC}$ in $g_{\ell}$. The function $f_{\ell}$ does not have a BC in the complex- $\omega$ plane [2, 34, and so the BC of $G_{\ell}\left(r, r^{\prime} ; \omega\right)$ along the NIA is due to the corresponding BC in $g_{\ell}$. Note that the Wronskian $W(\omega)$, and therefore also $A_{\ell, \omega}^{i n}$, inherits the BC on the NIA from $g_{\ell}$ (see, e.g., Eq.128 [34] and Eq.34 [2]).

It is convenient to define $\nu \equiv i \omega \in \mathbb{C}$ (and $\bar{\nu} \equiv \nu r_{h}$ ), which is positive along the NIA. We also define $\Delta A(-i \nu) \equiv$ $A_{+}(-i \nu)-A_{-}(-i \nu)$ for any function $A=A(\omega)$ possessing a BC along the NIA, where $A_{ \pm}(-i \nu) \equiv \lim _{\epsilon \rightarrow 0^{+}} A( \pm \epsilon-i \nu)$, with $\nu>0$. That is, $\Delta A$ is the discontinuity of $A(\omega)$ across the NIA.

As mentioned above, $f_{\ell}$ has poles on the NIA; these lie at $\bar{\nu}=k / 2, \forall k \in \mathbb{N}$ (with the exception for $s=2$ of the algebraically-special frequency $\bar{\nu}=\bar{\nu}_{A S}$, defined below, which is not a pole of $f_{\ell}$ [7, 9]), and so it is convenient to define a new radial function: $\hat{f}_{\ell}(r,-i \nu) \equiv-\sin (2 \pi \bar{\nu}) f_{\ell}(r,-i \nu)$. Accordingly, we define the Wronskian $\hat{W}(\omega) \equiv$ $W\left[g_{\ell}(r, \omega), \hat{f}_{\ell}(r, \omega)\right]$.

From the radial ODE Eq.2.2 and the boundary conditions Eq. 2.3), there follow the symmetries

$$
\begin{aligned}
g_{\ell}(r, \omega) & =g_{\ell}^{*}\left(r,-\omega^{*}\right) \quad f_{\ell}(r, \omega)=f_{\ell}^{*}\left(r,-\omega^{*}\right) \quad \text { if } \quad r_{*} \in \mathbb{R}, \\
W(\omega) & =W^{*}\left(-\omega^{*}\right), \quad \forall \omega \in \mathbb{C}
\end{aligned}
$$

These symmetries lead to

$$
\begin{aligned}
g_{\ell-}(r,-i \nu) & =g_{\ell+}^{*}(r,-i \nu) \quad \text { if } \quad r_{*} \in \mathbb{R}, \\
W_{-}(-i \nu) & =W_{+}^{*}(-i \nu), \quad \forall \nu>0,
\end{aligned}
$$

so that the discontinuity of $g_{\ell}$ across the NIA is only in its imaginary part. Note also that although $A_{\ell, \omega}^{i n}$ has a cut, $\left|A_{\ell, \omega}^{i n}\right|$ does not have a cut.

The BC contribution to the 'retarded' Green function is given by

$$
G^{B C}\left(x, x^{\prime}\right)=\sum_{\ell=0}^{\infty}(2 \ell+1) P_{\ell}(\cos \gamma) G_{\ell}^{B C}\left(r, r^{\prime} ; \Delta t\right), \quad G_{\ell}^{B C}\left(r, r^{\prime} ; \Delta t\right) \equiv \frac{1}{2 \pi i} \int_{0}^{\infty} d \nu \Delta G_{\ell}\left(r, r^{\prime} ;-i \nu\right) e^{-\nu \Delta t},
$$

The functions $g_{\ell+}$ and $g_{\ell-}$ satisfy the same homogeneous, linear 2nd order differential equation (namely, Eq. 2.2p). Therefore, $\Delta g_{\ell}$ will also satisfy this same differential equation and it may be expressed as a linear combination of the solutions $g_{\ell}(r,-i \nu)$ and $g_{\ell}(r,+i \nu)$. In addition, both $g_{\ell+}$ and $g_{\ell-}$ satisfy the same boundary condition (2.3) as $r \rightarrow \infty: g_{\ell \pm}(r,-i \nu) \sim e^{\nu r_{*}}$, and so $\Delta g_{\ell}$ will not satisfy this boundary condition. To understand this, note that the behaviour for $r \rightarrow \infty$ of $g_{\ell \pm}(r,-i \nu) \sim e^{\nu r_{*}}$ is dominant over that of $g_{\ell}(r,+i \nu) \sim e^{-\nu r_{*}}$, in $\Delta g_{\ell}$ the boundary conditions determine that the dominant terms cancel so that $\Delta g_{\ell}$ must be proportional to $g_{\ell}(r,+i \nu)$. Finally, $\Delta g_{\ell}$ must be purely-imaginary from Eq. 2.6] and, since we know that $g_{\ell}(r,+i \nu)$ is real-valued, it must be [2, 7] that

$$
\Delta g_{\ell}(r,-i \nu)=i q(\nu) g_{\ell}(r,+i \nu), \quad \forall \nu>0
$$

for some real-valued function $q(\nu)$ which entirely characterises the $\mathrm{BC}$ 'strength'.

Using Eq. 2.8), we can express the BC modes as [2, 7]

$$
\Delta G_{\ell}\left(r, r^{\prime} ;-i \nu\right)=-2 i \nu q(\nu) \frac{f_{\ell}(r,-i \nu) f_{\ell}\left(r^{\prime},-i \nu\right)}{W_{+}(-i \nu) W_{-}(-i \nu)}=-2 i \nu q(\nu) \frac{f_{\ell}(r,-i \nu) f_{\ell}\left(r^{\prime},-i \nu\right)}{\left|W_{ \pm}(-i \nu)\right|^{2}}, \quad \forall r_{*}, r_{*}^{\prime} \in \mathbb{R}
$$

The second step is due to Eq. 2.6, and we include the double subindex in $W_{ \pm}$because $\left|A_{\ell, \omega}^{i n}\right|$ has no cut. 


\section{LARGE- $\nu$ ASYMPTOTICS ON THE BRANCH CUT}

\section{A. Method}

We essentially follow the method of Maassen van den Brink [10, who calculates the high-frequency asymptotics of $f_{\ell}, g_{\ell+}$ and $q(\nu)$ on the NIA for the case $s=2$, to obtain the corresponding asymptotics for the cases $s=0$ and 1 . For completeness, we will also include the results of [10] for $s=2$. We now give a general description of the method.

As described in the previous section it is convenient to impose the boundary conditions on $g_{\ell}$ and $f_{\ell}$ in the complex $r$ or $r_{*}$ plane. The relationship between these two planes is complicated by the existence of a cut in the interrelationship $r_{*}=r+r_{h} \ln \left(r / r_{h}-1\right)$. Of special importance to our analysis are the anti-Stokes lines which for $\omega$ on the NIA are defined as the curves on the complex- $r$ plane where $\operatorname{Re}\left(r_{*}\right)=0$. In this case there are four anti-Stokes lines emanating from near $r=0$ and they have slopes equal to \pm 1 (i.e., $\arg r= \pm \pi / 4, \pm 3 \pi / 4$ ). The full structure is illustrated in Fig.1(a). We have two linearly-independent WKB asymptotic expansions for large- $\bar{\nu}, g_{a}(r, \mp i \nu) \sim e^{ \pm \nu r_{*}} ;$ these expansions are valid for $|\bar{r} \sqrt{\bar{\nu}}| \gg 1$ and away from the singularities $r=0$ and $r_{h}$ of the radial ODE. The importance of the anti-Stokes lines is that along them neither expansion, $g_{a}(r,+i \nu)$ nor $g_{a}(r,-i \nu)$, dominates over the other.

The contour chosen for $g_{\ell}(r,-i \nu)$, illustrated in Fig $1(\mathrm{~b})$, is as follows. By analytic continuation, we can impose the boundary condition for $g_{\ell}(r,-i \nu)$ on the anti-Stokes line going to $|r| \rightarrow \infty$ on the upper complex- $r$ plane instead of imposing it for $r \rightarrow \infty$ 32, 33. One can safely match $g_{\ell}(r,-i \nu)$ to $g_{a}(r,-i \nu)$ for $|r| \rightarrow \infty$ on that anti-Stokes line (i.e., at point A of Fig, $1(\mathrm{~b}))$ since, there, $g_{a}(r,-i \nu)$ does not dominate over $g_{a}(r,+i \nu)$. One can can then continue this expression from $|r| \rightarrow \infty$ along the anti-Stokes line down to a region near $r=0$ with $\arg r=3 \pi / 4$. (i.e., down to point B of Fig, 1(b)). However, one cannot continue this expression from point B to $r>r_{h}$ since one would have to cross into a region where $g_{a}(r,-i \nu)$ dominates over $g_{a}(r,+i \nu)$. Therefore, instead we introduce two linearly-independent solutions $\psi_{i}, i=1,2$, whose behaviour we can determine analytically and which we can match to the solutions we are interested in. Of course, we cannot determine these solutions exactly but we can obtain them in the limit of large $\bar{\nu}$ with fixed $\bar{r} \sqrt{\bar{\nu}}$ and this is sufficient for our purpose. In this approximation, the solutions $\psi_{i}$ are expressed in terms of special functions which one knows how to analytically continue from one anti-Stokes line to another. One then matches the solutions $\psi_{i}$ to $g_{a}(r,-i \nu) \sim g_{\ell+}(r,-i \nu)$ along $\arg r=3 \pi / 4$ in a region of overlap, given by $\bar{\nu}^{-1 / 2} \ll \bar{r} \ll \bar{\nu}^{-1 / 3}$ (a region which includes point B of Fig $1(\mathrm{~b})$ ). Since one knows how to analytically continue $\psi_{i}$ around $r=0$, with the matching done, one can analytically-continue $g_{\ell+}(r,-i \nu)$ from the anti-Stokes line on $\arg r=3 \pi / 4$ to another anti-Stokes line on $\arg r=\pi / 4$ and have it expressed as a linear combination of $g_{a}(r, \pm i \nu)$. This linear combination is safely valid along the anti-Stokes line clockwise all the way to $r_{*}=0$ (i.e., point $\mathrm{C}$ of Fig 1 (b)). The coefficient of $g_{a}(r,-i \nu)$ in this linear combination is 1 , and the coefficient of $g_{a}(r,+i \nu)$ yields the asymptotics for the BC 'strength' $q(\nu)$. From $r_{*}=0$, the linear combination is asymptotically valid on the physical line, either along $r_{*} \in(-\infty, 0)$ or along $r_{*} \in(0, \infty)$, even though the coefficient of the subdominant solution in the corresponding region becomes meaningless.

One can also find, using Frobenius method, two linearly-independent series expansions, $\psi_{1}^{F}(r)$ and $\psi_{2}^{F}(r)$, about $r=0$. These expansions are valid for $\left|\bar{r}^{2} \bar{\nu}\right| \ll 1$ and so there is no overlap with the region of validity of $g_{a}(r, \pm i \nu)$; the expansions $\psi_{i}^{F}$ do however provide a check for the solutions $\psi_{i}$.

The contour chosen for $f_{\ell}(r,-i \nu)$, illustrated in Fig 1 (c), is as follows. First, from its boundary condition as $r_{*} \rightarrow-\infty$ we know that at $r_{*}=0$ the solution $f_{\ell}(r,-i \nu)$ has to be a linear combination of $g_{a}(r, \pm i \nu)$ with the coefficient of $g_{a}(r,+i \nu)$ (which is the dominant WKB expansion on $r_{*} \in(-\infty, 0)$ ) being equal to 1 . This linear combination of $g_{a}(r, \pm i \nu)$ is valid anticlockwise along the anti-Stokes line from $r_{*}=0$ up to arg $r=\pi / 4$. In order to obtain the coefficient of $g_{a}(r,-i \nu)$, one re-expresses this linear combination as a linear combination of $\psi_{1}$ and $\psi_{2}$, on $\arg r=\pi / 4$, using the matching described above. One knows how to analytically continue this expression onto the anti-Stokes line on $\arg r=-\pi / 4$, and there it can be re-expressed as a new linear combination of $g_{a}(r, \pm i \nu)$. One can then continue this linear combination anticlockwise along the anti-Stokes line back to $r_{*}=0$, thus yielding the asymptotic monodromy of $f_{\ell}$ around $r=r_{h}$. The obtained asymptotic monodromy is then compared with the exact monodromy $f_{\ell}\left(\left(r-r_{h}\right) e^{2 \pi i},-i \nu\right)=e^{-2 \pi i \bar{\nu}} f_{\ell}\left(r-r_{h},-i \nu\right)$, which follows from the boundary condition on $f_{\ell}$ at the horizon. The comparison then yields the coefficient of $g_{a}(r,-i \nu)$ that we wanted.

Let us note that in 32 they use a similar, but different, method to the one we use here. In 32 they make use of the Stokes phenomenon, instead of the solutions $\psi_{i}$, in order to continue from one anti-Stokes line to another. For that reason, they require the knowledge of the topology of the anti-Stokes lines very near $r=0$, down to $r=O\left(\bar{\nu}^{-1 / 2}\right)$, where their WKB expansion breaks down - see Fig.1 32]. In our analysis, however, the WKB expansions never reach a region so near $r=0$. See also [11 for a description of similar contours followed. 

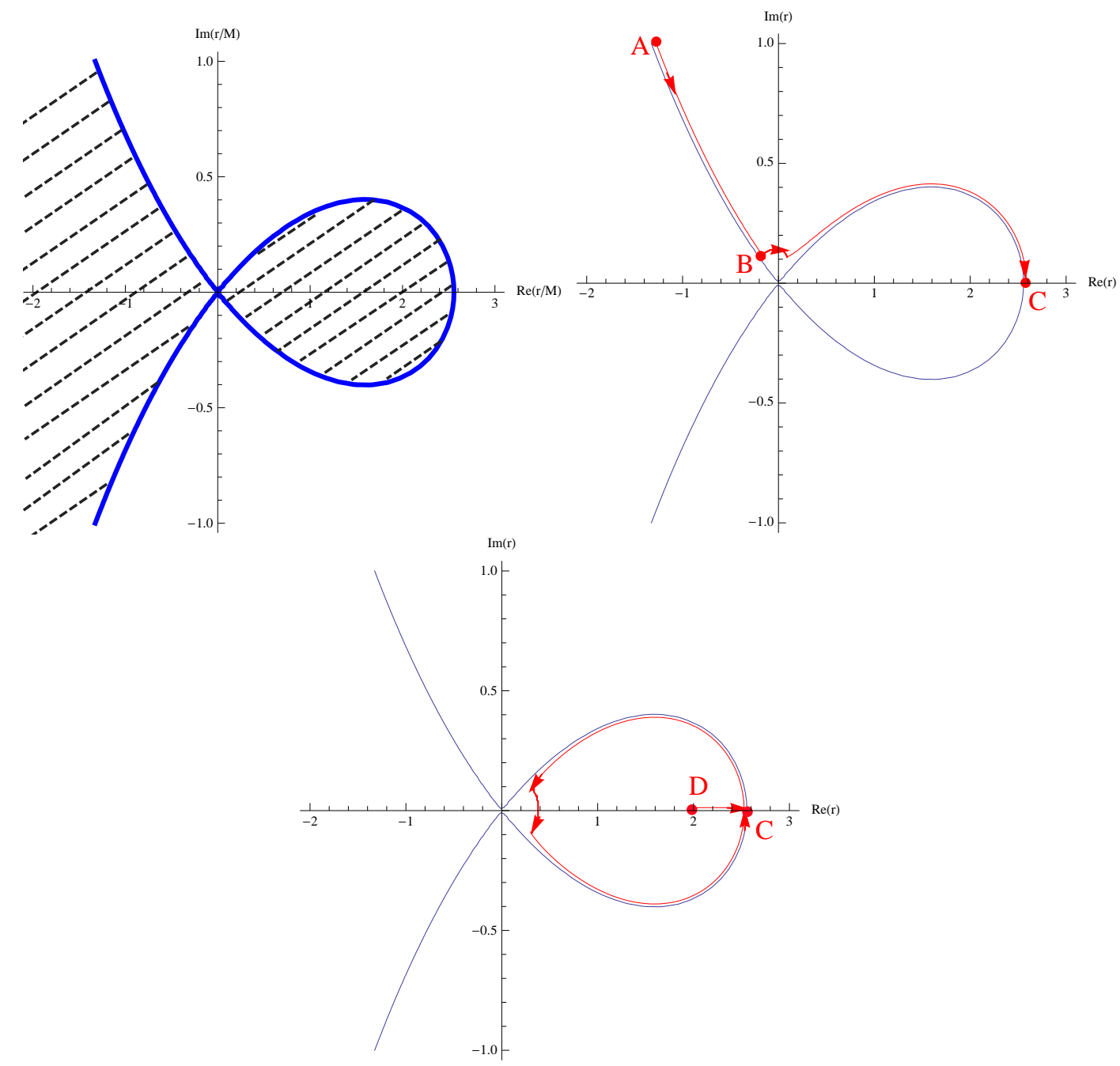

FIG. 1: (a) In blue: schematic illustration of anti-Stokes lines (i.e., where $\operatorname{Re}\left(r_{*}\right)=0$ ) on the complex- $r$ plane in the case that $\omega$ is on the NIA in Schwarzschild. [See, e.g., Fig.1 [32] for the topology of their anti-Stokes lines for large-frequency very near $r=0$, where their method differs only slightly from the standard WKB analysis.] In dashed black lines: the region where $\operatorname{Re}\left(r_{*}\right)<0$. (b) In red: the contour we follow in order to calculate the large- $\bar{\nu}$ asymptotics for $g_{\ell+}$. (c) In red: the contour followed in order to calculate $f_{\ell}$. Point A corresponds to a point for $|\bar{r}| \gg 1$ along the anti-Stokes line that stems from near $r=0$ at $\arg (r)=3 \pi / 4$; point $\mathrm{B}$ corresponds to a point on that same anti-Stokes line but lies within $\bar{\nu}^{-1 / 2} \ll \bar{r} \ll \bar{\nu}^{-1 / 3} ;$ point $\mathrm{C}$ corresponds to $r_{*}=0$; point $\mathrm{D}$ corresponds to a point with $r \gtrsim r_{h}$ but $r$ sufficiently far from $r_{h}$ so that the WKB expansions $g_{a}(r, \pm i \nu)$ are valid.

\section{B. Radial solution $g_{\ell}(r,-i \nu)$}

Firstly, we find WKB asymptotic expansions for the solution of the radial ODE for $|\bar{\omega}| \gg 1$ :

$$
\begin{aligned}
& g_{a}(r, \omega) \equiv e^{i \omega r_{*}}\left\{1+\frac{g_{1}(r)}{\bar{\omega}}+\frac{g_{2}(r)}{\bar{\omega}^{2}}+\ldots\right\} \\
& g_{1}(r)=\frac{1}{2 i} \int_{\infty}^{r} d v \frac{v V(v)}{v-1}=\frac{i\left[2 \lambda \bar{r}+1-s^{2}\right]}{4 \bar{r}^{2}} \\
& g_{2}(r)=\frac{V(r)}{4}-\frac{1}{8}\left[\int_{\infty}^{r} d v \frac{v V(v)}{v-1}\right]^{2}=\frac{8(\bar{r}-1)\left[\lambda \bar{r}+1-s^{2}\right]-\left[2 \lambda \bar{r}+1-s^{2}\right]^{2}}{32 \bar{r}^{4}}
\end{aligned}
$$


These expansions $g_{a}(r, \omega)$ are valid away from the singularities $r=0$ (specifically, $|\bar{r} \sqrt{\bar{\nu}}| \gg 1$ is required) and $r_{h}$ of the radial ODE and, they are such that neither expansion, $g_{a}(r,+i \nu)$ nor $g_{a}(r,-i \nu)$, dominates over the other along an anti-Stokes line.

Secondly, we use the Frobenius method to find two linearly-independent solutions about $r=0$ of the radial ODE (re-expressed with $r$, not $r_{*}$, as the independent variable). The characteristic exponents are: $1 \pm s$, and so they differ by an integer number when $s=0,1,2$. We first find a power series solution $\psi_{1}^{F}(r)$ about $\bar{r}=0$ :

$$
\begin{array}{ll}
s=0: & \psi_{1}^{F}(r)=\bar{r}-\lambda \bar{r}^{2}+O\left(\bar{r}^{3}\right) \\
s=1: & \psi_{1}^{F}(r)=\bar{r}^{2}-\frac{(\lambda-2)}{3} \bar{r}^{3}+\frac{(\lambda-2)(\lambda-6)}{24} \bar{r}^{4}+O\left(\bar{r}^{5}\right) \\
s=2: & \psi_{1}^{F}(r)=\bar{r}^{3}-\frac{(\lambda-6)}{5} \bar{r}^{4}+O\left(\bar{r}^{5}\right)
\end{array}
$$

and the second, linearly independent solution $\psi_{2}^{F}(r)$ is given by

$$
\begin{array}{ll}
s=0: & \psi_{2}^{F}(r)=[1+2 \lambda] \bar{r}^{2}+\frac{\left(2+2 \lambda-3 \lambda^{2}\right)}{4} \bar{r}^{3}+O\left(\bar{r}^{4}\right)+\psi_{1}^{F}(r) \ln \bar{r} \\
s=1: & \psi_{2}^{F}(r)=1+\lambda \bar{r}+O\left(\bar{r}^{2}\right)-\frac{\lambda^{2}}{2} \psi_{1}^{F}(r) \ln \bar{r} \\
s=2: & \psi_{2}^{F}(r)=\frac{1}{\bar{r}}+\frac{2 \nu_{0}}{3}+\frac{\bar{\nu}_{A S}}{2} \bar{r}+O\left(\bar{r}^{2}\right)+\frac{\left(\bar{\nu}^{2}-\bar{\nu}_{A S}^{2}\right)}{4} \psi_{1}^{F}(r) \ln \bar{r}
\end{array}
$$

where $\bar{\nu}=\bar{\nu}_{A S} \equiv \lambda(\lambda-2) / 6$ is the so-called algebraically special frequency (note that we use this term to refer both to $\bar{\nu}_{A S}$ and to $\left.\omega_{A S}=-i \nu_{A S}\right)$. Note the exact monodromies:

$$
\begin{array}{ll}
s=0: & \psi_{2}^{F}\left(r e^{2 \pi i}\right)=\psi_{2}^{F}(r)+2 \pi i \psi_{1}^{F}(r) \\
s=1: & \psi_{2}^{F}\left(r e^{2 \pi i}\right)=\psi_{2}^{F}(r)-\lambda^{2} \pi i \psi_{1}^{F}(r) \\
s=2: & \psi_{2}^{F}\left(r e^{2 \pi i}\right)=\psi_{2}^{F}(r)+\frac{\left(\bar{\nu}^{2}-\bar{\nu}_{A S}^{2}\right) \pi i}{2} \psi_{1}^{F}(r)
\end{array}
$$

and $\psi_{1}^{F}\left(r e^{2 \pi i}\right)=\psi_{1}^{F}(r), \forall s=0,1,2$.

We now rewrite the ODE in terms of the independent variable $t \equiv \bar{r} \sqrt{\bar{\nu}}$ and then group terms in different powers of $\bar{\nu}$ :

$$
\begin{aligned}
& \hat{L}_{0} \psi=\frac{1}{\sqrt{\bar{\nu}}} \hat{L}_{1} \psi+\frac{1}{\bar{\nu}} \hat{L}_{2} \psi \\
& \hat{L}_{0} \equiv t^{2} \frac{d^{2}}{d t^{2}}-t \frac{d}{d t}-\left(s^{2}-1\right)-t^{4}, \quad \hat{L}_{1} \equiv 2 t^{3} \frac{d^{2}}{d t^{2}}-t^{2} \frac{d}{d t}-\left[\lambda+s^{2}-1\right] t, \quad \hat{L}_{2} \equiv-t^{4} \frac{d^{2}}{d t^{2}}+\lambda t^{2}
\end{aligned}
$$

Expanding for large- $\bar{\nu}$, there are two linearly-independent solutions $\psi_{i}=\psi_{i}^{(0)}+\psi_{i}^{(1)}, i=1,2$, where $\psi_{i}^{(0)}$ denotes the two leading order solutions and $\psi_{i}^{(1)}$ the next-to-leading order ones:

$$
\begin{aligned}
\hat{L}_{0} \psi_{i}^{(0)}(t) & =0, \quad \psi_{i}^{(1)}(t)=\int_{0}^{t} d u G_{\psi}(t, u) R_{i}(u), \quad R_{i}(t) \equiv \frac{1}{\sqrt{\bar{\nu}}} \hat{L}_{1} \psi_{i}^{(0)}(t), \quad \forall i=1,2 \\
G_{\psi}(t, u) & \equiv \frac{\left[-\psi_{1}^{(0)}(t) \psi_{2}^{(0)}(u)+\psi_{2}^{(0)}(t) \psi_{1}^{(0)}(u)\right]}{u^{2} W\left[\psi_{1}^{(0)}(u), \psi_{2}^{(0)}(u)\right]}, \quad W\left[\psi_{1}^{(0)}(u), \psi_{2}^{(0)}(u)\right] \equiv \psi_{1}^{(0)}(u) \frac{d \psi_{2}^{(0)}(u)}{d u}-\psi_{2}^{(0)}(u) \frac{d \psi_{1}^{(0)}(u)}{d u}
\end{aligned}
$$

This order will be sufficient for $s=0$ and 2. However, for $s=1$ we will require the next order, $\psi_{i}=\psi_{i}^{(0)}+\psi_{i}^{(1)}+\psi_{i}^{(2)}$, with:

$$
\begin{aligned}
\psi_{i}^{(2)}(t) & =\psi_{i}^{(2 a)}(t)+\psi_{i}^{(2 b)}(t), \quad i=1,2 \\
\psi_{i}^{(2 a)}(t) & =\int_{0}^{t} d u G_{\psi}(t, u) R_{i}^{(1 a)}(u), \quad R_{i}^{(1 a)}(t) \equiv \frac{1}{\bar{\nu}} \hat{L}_{2} \psi_{i}^{(0)}(t) \\
\psi_{i}^{(2 b)}(t) & =\int_{0}^{t} d u G_{\psi}(t, u) R_{i}^{(1 b)}(u), \quad R_{i}^{(1 b)}(t) \equiv \frac{1}{\sqrt{\bar{\nu}}} \hat{L}_{1} \psi_{i}^{(1)}(t)
\end{aligned}
$$


The leading order solutions can be found to be given by

$$
\begin{array}{ll}
s=0: & \psi_{1}^{(0)}(t)=\frac{t}{\sqrt{\bar{\nu}}} J_{0}\left(\frac{t^{2}}{2 i}\right), \quad \psi_{2}^{(0)}(t)=\frac{\pi t}{4 \sqrt{\bar{\nu}}} Y_{0}\left(\frac{t^{2}}{2 i}\right) \\
s=1: & \psi_{1}^{(0)}(t)=\frac{e^{-\pi i / 4} \sqrt{\pi}}{\bar{\nu}} t J_{1 / 2}\left(\frac{t^{2}}{2 i}\right)=\frac{2 \sinh \left(t^{2} / 2\right)}{\bar{\nu}}, \quad \psi_{2}^{(0)}(t)=\frac{e^{3 \pi i / 4} \sqrt{\pi}}{2} t Y_{1 / 2}\left(\frac{t^{2}}{2 i}\right)=\cosh \left(t^{2} / 2\right) \\
s=2: & \psi_{1}^{(0)}(t)=\frac{4 i t}{\bar{\nu}^{3 / 2}} J_{1}\left(\frac{t^{2}}{2 i}\right), \quad \psi_{2}^{(0)}(t)=\frac{i \pi \bar{\nu}^{1 / 2} t}{4} Y_{1}\left(\frac{t^{2}}{2 i}\right)
\end{array}
$$

We have chosen the normalization constants so that, as indicated below, these solutions agree with the corresponding solutions $\psi_{i}^{F}$. As for the quantities in the next-to-leading order solutions (and also the following order for $s=1$ ), we find the following. For $s=0$,

$$
\begin{aligned}
& W\left[\psi_{1}^{(0)}(u), \psi_{2}^{(0)}(u)\right]=\frac{u}{\bar{\nu}} \\
& R_{1}(t)=\frac{t^{2}\left[i t^{2} J_{1}\left(\frac{t^{2}}{2 i}\right)-\left(\lambda-2 t^{4}\right) J_{0}\left(\frac{t^{2}}{2 i}\right)\right]}{\bar{\nu}} \\
& R_{2}(t)=\frac{\pi t^{2}\left[i t^{2} Y_{1}\left(\frac{t^{2}}{2 i}\right)-\left(\lambda-2 t^{4}\right) Y_{0}\left(\frac{t^{2}}{2 i}\right)\right]}{4 \bar{\nu}}
\end{aligned}
$$

For $s=1$,

$$
\begin{aligned}
& W\left[\psi_{1}^{(0)}(u), \psi_{2}^{(0)}(u)\right]=-\frac{2 u}{\bar{\nu}} \\
& R_{1}(t)=\frac{2 t\left[t^{2} \cosh \left(t^{2} / 2\right)-\left(\lambda-2 t^{4}\right) \sinh \left(t^{2} / 2\right)\right]}{\bar{\nu}^{3 / 2}} \\
& \psi_{1}^{(1)}(t)=-\frac{4 t^{3} \cosh \left(t^{2} / 2\right)+3 \sqrt{\pi} \lambda\left[e^{-t^{2} / 2} \operatorname{erfi}(t)-\operatorname{erf}(t) e^{t^{2} / 2}\right]}{6 \bar{\nu}^{3 / 2}} \\
& R_{2}(t)=\frac{t\left[t^{2} \sinh \left(t^{2} / 2\right)-\left(\lambda-2 t^{4}\right) \cosh \left(t^{2} / 2\right)\right]}{\bar{\nu}^{1 / 2}} \\
& \psi_{2}^{(1)}(t)=-\psi_{1}^{(0)}(t)\left\{\int_{0}^{t} \frac{d u}{u^{2}}\left[\frac{\psi_{2}^{(0)}(u) R_{2}(u)}{W\left[\psi_{1}^{(0)}(u), \psi_{2}^{(0)}(u)\right]}-\frac{\sqrt{\bar{\nu}} \lambda}{2}\right]-\frac{\sqrt{\bar{\nu}} \lambda}{2 t}\right\}+\psi_{2}^{(0)}(t) \int_{0}^{t} \frac{d u}{u^{2}} \frac{\psi_{1}^{(0)}(u) R_{2}(u)}{W\left[\psi_{1}^{(0)}(u), \psi_{2}^{(0)}(u)\right]}= \\
& \frac{12 \lambda \sinh \left(t^{2} / 2\right)+e^{-t^{2} / 2}\left\{2\left(e^{t^{2} / 2}-1\right)\left[t^{4}-3 \lambda\right]+3 \lambda \sqrt{\pi} t\left[e^{t^{2}} \operatorname{erf}(t)+\operatorname{erfi}(t)\right]\right\}}{12 t \bar{\nu}^{1 / 2}},
\end{aligned}
$$

For $s=1$, we also required the following order. The first solution for $s=1$ to following order is given by

$$
\begin{aligned}
& R_{1}^{(1 a)}(t)=\frac{2 t^{2}\left[-t^{2} \cosh \left(t^{2} / 2\right)+\left(\lambda-t^{4}\right) \sinh \left(t^{2} / 2\right)\right]}{\bar{\nu}^{2}} \\
& R_{1}^{(1 b)}(t)=\frac{e^{-\frac{t^{2}}{2}} t \sqrt{\pi} \ell\left\{(\ell+1) e^{t^{2}} \operatorname{erf}(t)\left(\lambda-2 t^{4}-t^{2}\right)-\operatorname{erfi}(t)\left[\ell^{3}+2 \ell^{2}+\ell\left(-2 t^{4}+t^{2}+1\right)-2 t^{4}+t^{2}\right]\right\}}{2 \bar{\nu}^{2}}- \\
& \frac{e^{-\frac{t^{2}}{2}} t^{2}\left\{\lambda\left[t^{2}+e^{t^{2}}\left(t^{2}+3\right)-3\right]-t^{2}\left[2 t^{4}-13 t^{2}+e^{t^{2}}\left(2 t^{4}+13 t^{2}+9\right)+9\right]\right\}}{3 \bar{\nu}^{2}} \\
& \psi_{1}^{(2 a)}(t)=\frac{-\cosh \left(t^{2} / 2\right)\left\{-2 \gamma_{E} \lambda+t^{4}+2 \lambda\left[\operatorname{chi}\left(t^{2}\right)-2 \ln t\right]\right\}+2 \lambda \sinh \left(t^{2} / 2\right) \operatorname{shi}\left(t^{2}\right)}{4 \bar{\nu}^{2}}
\end{aligned}
$$


while the second solution is given by

$$
\begin{aligned}
& R_{2}^{(1 a)}(t)=\frac{t^{2}\left[\left(\lambda-t^{4}\right) \cosh \left(t^{2} / 2\right)-t^{2} \sinh \left(t^{2} / 2\right)\right]}{\bar{\nu}} \\
& R_{2}^{(1 b)}(t)=\frac{-e^{-\frac{t^{2}}{2}} t \sqrt{\pi} \ell\left\{(\ell+1) e^{t^{2}} \operatorname{erf}(t)\left(\ell^{2}+\ell-2 t^{4}-t^{2}\right)+\operatorname{erfi}(t)\left[\ell^{3}+2 \ell^{2}+\ell\left(-2 t^{4}+t^{2}+1\right)-2 t^{4}+t^{2}\right]\right\}}{4 \bar{\nu}}- \\
& \frac{e^{-\frac{t^{2}}{2}} t^{2}\left\{\lambda\left[-t^{2}+e^{t^{2}}\left(t^{2}+3\right)+3\right]-t^{2}\left[-2 t^{4}+13 t^{2}+e^{t^{2}}\left(2 t^{4}+13 t^{2}+9\right)-9\right]\right\}}{6 \bar{\nu}} \\
& \psi_{2}^{(2 a)}(t)=-\psi_{1}^{(0)}(t)\left\{\int_{0}^{t} \frac{d u}{u}\left[\frac{\psi_{2}^{(0)}(u) R_{2}^{(1 a)}(u)}{u W\left[\psi_{1}^{(0)}(u), \psi_{2}^{(0)}(u)\right]}+\frac{\lambda}{2}\right]-\frac{\lambda}{2} \ln t\right\}+\psi_{2}^{(0)}(t) \int_{0}^{t} \frac{d u}{u^{2}} \frac{\psi_{1}^{(0)}(u) R_{2}^{(1 a)}(u)}{W\left[\psi_{1}^{(0)}(u), \psi_{2}^{(0)}(u)\right]} \\
& \psi_{2}^{(2 b)}(t)=-\psi_{1}^{(0)}(t)\left\{\int_{0}^{t} \frac{d u}{u}\left[\frac{\psi_{2}^{(0)}(u) R_{2}^{(1 b)}(u)}{u W\left[\psi_{1}^{(0)}(u), \psi_{2}^{(0)}(u)\right]}-\frac{\lambda(\lambda+1)}{2}\right]+\frac{(\lambda+1) \lambda}{2} \ln t\right\}+ \\
& \psi_{2}^{(0)}(t) \int_{0}^{t} \frac{d u}{u^{2}} \frac{\psi_{1}^{(0)}(u) R_{2}^{(1 b)}(u)}{W\left[\psi_{1}^{(0)}(u), \psi_{2}^{(0)}(u)\right]}
\end{aligned}
$$

where $\operatorname{erf}(z)$ and $\operatorname{erfi}(z)=\operatorname{erf}(i z) / i$ are, respectively, the error function and the imaginary error function, and $\operatorname{chi}(z)$ and $\operatorname{shi}(z)$ are, respectively, the hyperbolic cosine integral and the hyperbolic sine integral functions. Note that while $\operatorname{shi}(z)$ is an entire function of $z \in \mathbb{C}, \operatorname{chi}(z)$ has a branch cut along the negative real axis. Finally, the next-to-leading order solutions for $s=2$ are given by

$$
\begin{aligned}
& W\left[\psi_{1}^{(0)}(u), \psi_{2}^{(0)}(u)\right]=-\frac{4 u}{\bar{\nu}} \\
& \psi_{1}^{(1)}(t)=\frac{\pi}{4} t \int_{0}^{t} \frac{d u}{u^{2}}\left[Y_{1}\left(\frac{t^{2}}{2 i}\right) J_{1}\left(\frac{u^{2}}{2 i}\right)-J_{1}\left(\frac{t^{2}}{2 i}\right) Y_{1}\left(\frac{u^{2}}{2 i}\right)\right] R_{1}(u), \\
& R_{1}(u)=\frac{4 i}{\bar{\nu}^{2}}\left\{2\left[u^{6}-\nu_{0} u^{2}\right] J_{1}\left(\frac{u^{2}}{2 i}\right)-i u^{4} J_{0}\left(\frac{u^{2}}{2 i}\right)\right\} \\
& \psi_{2}^{(1)}(t)=\frac{\pi}{4} t Y_{1}\left(\frac{t^{2}}{2 i}\right) \int_{0}^{t} \frac{d u}{u^{2}} J_{1}\left(\frac{u^{2}}{2 i}\right) R_{2}(s)+\frac{\pi}{4} t J_{1}\left(\frac{t^{2}}{2 i}\right)\left\{\int_{0}^{t} d u\left[\frac{8 i \nu_{0}}{\pi u^{4}}-Y_{1}\left(\frac{u^{2}}{2 i}\right) \frac{R_{2}(u)}{u^{2}}\right]+\frac{8 i \nu_{0}}{3 \pi t^{3}}\right\}, \\
& R_{2}(u)=\frac{i \pi}{4}\left\{2\left[u^{6}-\nu_{0} s^{2}\right] Y_{1}\left(\frac{u^{2}}{2 i}\right)-i u^{4} Y_{0}\left(\frac{u^{2}}{2 i}\right)\right\}
\end{aligned}
$$

Note that the integrand for $\psi_{2}^{(1)}$ given by Eq. 3.6. leads to an integral divergent at the lower limit, both for $s=1$ and $s=2$. We therefore regularize it by integrating by parts and dropping the contribution at the lower limit which corresponds to adding an (infinite) multiple of the leading-order first solution, $\psi_{1}^{(0)}$. A similar divergence occurs for $\psi_{2}^{(2 a)}$ and $\psi_{2}^{(2 b)}$ for $s=1$, and we regularize them similarly. One can check that by taking an expansion as $t \rightarrow 0$ for $\psi_{i}^{(0)}(t)+\psi_{i}^{(1)}(t), i=1,2$, both the leading order and the next-to-leading-order terms as $r \rightarrow 0$ in Eqs. 3.2 and 3.3. are recovered for $s=0,1$ and 2. In the $s=1$ case we have also checked that the leading order term for $t \rightarrow 0$ of $\psi_{1}^{(2)}(t)$ recovers the $O\left(\bar{r}^{4}\right)$ term of $\psi_{1}^{F}(r)$ in Eq. 3.2); similarly, the leading order term for $t \rightarrow 0$ of $\psi_{2}^{(2)}(t)$ recovers the $O\left(\bar{r}^{4} \ln \bar{r}\right)$ term of $\psi_{2}^{F}(r)$ in Eq. $(3.3)$.

Using the formulae from [37,

$$
\begin{aligned}
& J_{\mu}\left(z e^{m \pi i}\right)=e^{m \mu \pi i} J_{\mu}(z) \\
& Y_{\mu}\left(z e^{m \pi i}\right)=e^{-m \mu \pi i} Y_{\mu}(z)+2 i \sin (m \mu \pi) \cot (\mu \pi) J_{\mu}(z), \\
& Y_{k}\left(z e^{m \pi i}\right)=(-1)^{m k}\left[Y_{k}(z)+2 i m J_{k}(z)\right],
\end{aligned}
$$

valid $\forall z, \mu \in \mathbb{C}$ and $\forall m, k \in \mathbb{Z}$, we obtain the following analytic continuations for the various terms in $\psi_{i}$. For $s=0$ :

$$
\begin{array}{ll}
\psi_{1}^{(0)}(i t)=i \psi_{1}^{(0)}(t), & \psi_{2}^{(0)}(i t)=i \psi_{2}^{(0)}(t)-\frac{\pi}{2} \psi_{1}^{(0)}(t), \\
\psi_{1}^{(1)}(i t)=-\psi_{1}^{(1)}(t), & \psi_{2}^{(1)}(i t)=-\psi_{2}^{(1)}(t)-\frac{\pi i}{2} \psi_{1}^{(1)}(t)
\end{array}
$$


For $s=1$ :

$$
\begin{array}{rlrl}
\psi_{1}^{(0)}(i t) & =-\psi_{1}^{(0)}(t), & \psi_{2}^{(0)}(i t) & =\psi_{2}^{(0)}(t), \\
\psi_{1}^{(1)}(i t) & =-i \psi_{1}^{(1)}(t), & \psi_{2}^{(1)}(i t) & =i \psi_{2}^{(1)}(t) \\
\psi_{1}^{(2 a)}(i t) & =\psi_{1}^{(2 a)}(t), & \psi_{2}^{(2 a)}(i t) & =-\psi_{2}^{(2 a)}(t)-\frac{\pi i \lambda}{4} \psi_{1}^{(0)}(t), \\
\psi_{1}^{(2 b)}(i t) & =\psi_{1}^{(2 b)}(t), & \psi_{2}^{(2 b)}(i t) & =-\psi_{2}^{(2 b)}(t)+\frac{\pi i \lambda(\lambda+1)}{4} \psi_{1}^{(0)}(t) \\
\psi_{2}^{(2)}(i t) & =-\psi_{2}^{(2)}(t)+A \psi_{1}^{(0)}(t), \quad A \equiv \frac{\pi i \lambda^{2}}{4}
\end{array}
$$

Note the terms with $\psi_{1}^{(0)}(t)$ in $\psi_{2}^{(2 a)}(i t), \psi_{2}^{(2 b)}(i t)$ and $\psi_{2}^{(2)}(i t)$ arising from the regularization terms.

For $s=2$ :

$$
\begin{array}{ll}
\psi_{1}^{(0)}(i t)=-i \psi_{1}^{(0)}(t), & \psi_{2}^{(0)}(i t)=-i \psi_{2}^{(0)}(t)+\frac{\pi \bar{\nu}^{2}}{8} \psi_{1}^{(0)}(t), \\
\psi_{1}^{(1)}(i t)=\psi_{1}^{(1)}(t), & \psi_{2}^{(1)}(i t)=\psi_{2}^{(1)}(t)+i \frac{\pi \bar{\nu}^{2}}{8} \psi_{1}^{(1)}(t)
\end{array}
$$

It is also easy to check that, along $\arg t=\pi / 4$, we have

$$
\begin{aligned}
s= & 0: \quad \psi_{1,2}^{(0)} e^{-\pi i / 4} \in \mathbb{R}, \quad \psi_{1,2}^{(1)} e^{-\pi i / 2} \in \mathbb{R} \\
s= & 1: \quad \psi_{1}^{(0)} e^{\pi i / 2}, \psi_{2}^{(0)} \in \mathbb{R}, \quad \psi_{1}^{(1)} e^{\pi i / 4}, \psi_{2}^{(1)} e^{-\pi i / 4} \in \mathbb{R}, \\
& \psi_{1}^{(2)} \in \mathbb{R}, \quad\left(\psi_{2}^{(2)}+\frac{A}{2} \psi_{1}^{(0)}\right) e^{\pi i / 2} \in \mathbb{R} \\
s= & 2: \quad \psi_{1,2}^{(0)} e^{-3 \pi i / 4} \in \mathbb{R}, \quad \psi_{1,2}^{(1)} \in \mathbb{R}
\end{aligned}
$$

Next, we carry out an asymptotic series expansion for large- $|t|$ along $\arg t=\pi / 4$ of $\psi_{i}^{(0)}$ and $\psi_{i}^{(1)}$ (and $\psi_{i}^{(2)}$ for $s=1$ ). In the corresponding expressions which we write next, we only show the $t^{0}$ terms in each of these large- $|t|$ expansions. Using Eqs.(3.18), we can then write for $s=0$ :

$$
\begin{array}{ll}
\psi_{1}(t) \sim \frac{1}{\sqrt{\pi \bar{\nu}}}\left\{e^{t^{2} / 2}\left[1+\frac{\alpha_{1}}{\sqrt{\bar{\nu}}}\right]+e^{-t^{2} / 2}\left[i-\frac{\alpha_{1}^{*}}{\sqrt{\bar{\nu}}}\right]\right\} & \\
\psi_{2}(t) \sim \frac{\sqrt{\pi}}{4 \sqrt{\bar{\nu}}}\left\{e^{t^{2} / 2}\left[-i+\frac{\beta_{1}}{\sqrt{\bar{\nu}}}\right]+e^{-t^{2} / 2}\left[-1-\frac{\beta_{1}^{*}}{\sqrt{\bar{\nu}}}\right]\right\}, \quad \arg t=\pi / 4
\end{array}
$$

for $s=1$ :

$$
\begin{array}{ll}
\psi_{1}(t) \sim \frac{1}{\bar{\nu}}\left\{e^{t^{2} / 2}\left[1+\frac{\alpha_{1}}{\bar{\nu}^{1 / 2}}+\frac{\alpha_{2}}{\bar{\nu}}\right]+e^{-t^{2} / 2}\left[-1-\frac{i \alpha_{1}^{*}}{\sqrt{\bar{\nu}}}+\frac{\alpha_{2}^{*}}{\bar{\nu}}\right]\right\} \\
\psi_{2}(t) \sim \frac{1}{2}\left\{e^{t^{2} / 2}\left[1+\frac{\beta_{1}}{\bar{\nu}^{1 / 2}}+\frac{\beta_{2}-A}{\bar{\nu}}\right]+e^{-t^{2} / 2}\left[1+\frac{i \beta_{1}^{*}}{\sqrt{\bar{\nu}}}+\frac{-\beta_{2}^{*}+A}{\bar{\nu}}\right]\right\}, \quad \arg t=\pi / 4
\end{array}
$$

and for $s=2$ :

$$
\begin{array}{ll}
\psi_{1}(t) \sim \frac{4}{\sqrt{\pi \bar{\nu}^{3}}}\left\{e^{t^{2} / 2}\left[1+\frac{\alpha_{1}}{\sqrt{\bar{\nu}}}\right]+e^{-t^{2} / 2}\left[-i+\frac{\alpha_{1}^{*}}{\sqrt{\bar{\nu}}}\right]+O\left(t^{-2}\right)+O\left(\frac{t^{3}}{\sqrt{\bar{\nu}}}\right)+O\left(\bar{\nu}^{-1}\right)\right\} & \\
\psi_{2}(t) \sim \frac{\sqrt{\pi \bar{\nu}}}{4}\left\{e^{t^{2} / 2}\left[-i+\frac{\beta_{1}}{\sqrt{\bar{\nu}}}\right]+e^{-t^{2} / 2}\left[1+\frac{\beta_{1}^{*}}{\sqrt{\bar{\nu}}}\right]+O\left(t^{-2}\right)+O\left(\frac{t^{3}}{\sqrt{\bar{\nu}}}\right)+O\left(\bar{\nu}^{-1}\right)\right\}, \quad \arg t=\pi / 4
\end{array}
$$

which is valid for $1 \ll t \ll \bar{\nu}^{1 / 6}$ We can obtain the corresponding asymptotics for $\psi_{1,2}(t)$ at $\arg t=3 \pi / 4$ by using Eqs.3.19)-3.21) along $\arg t=\pi / 4$ together with the analytic continuation Eqs.(3.15)-3.17). The dominant term of such asymptotics at $\arg t=3 \pi / 4$ (i.e., the term with $e^{t^{2} / 2}$ ) must agree with the corresponding term in $\psi_{1,2}(i t)$, obtained from Eqs.(3.19)-3.21) for $\arg t=\pi / 4$. Similarly, we can take a linear combination of the asymptotics for $\psi_{1,2}(t)$ at $\arg t=3 \pi / 4$ such that the dominant term is zero, and then require that the remaining, subdominant term 
(i.e., the term with $e^{-t^{2} / 2}$ ) agrees with the corresponding term in the linear combination of $\psi_{1}(i t)$ and $\psi_{2}(i t)$, which are obtained from Eqs.(3.19)-(3.21) for $\arg t=\pi / 4$. The result of these requirements is:

$$
\begin{aligned}
s=0 \& 2: & \alpha_{1} \in \mathbb{R}, \quad \beta_{1}=-(2+i) \alpha_{1} \\
s=1: & \alpha_{1}, \alpha_{2} \in \mathbb{R}, \quad \beta_{1}=-\alpha_{1}, \quad \operatorname{Im}\left(\beta_{2}\right)=\alpha_{1}^{2} .
\end{aligned}
$$

The actual values of $\alpha_{1}$ can be obtained by directly expanding $\psi_{1}^{(1)}(t)$ in Eq. 3.6 for large $|t|$ with $\arg t=\pi / 4$. We obtain

$$
\begin{array}{ll}
s=0: & \alpha_{1}=-\frac{\Gamma\left(\frac{1}{4}\right)^{4}}{48 \pi^{3 / 2}}[1+3 \lambda] \\
s=1: & \alpha_{1}=-\frac{\lambda \sqrt{\pi}}{2} \\
s=2: & \alpha_{1}=\frac{\Gamma\left(\frac{1}{4}\right)^{4}}{48 \pi^{3 / 2}}[1-\lambda]
\end{array}
$$

From the boundary condition Eq. 2.3) and the leading-order behaviour of Eq. (3.1) it follows that

$$
g_{\ell+}(r,-i \nu) \sim g_{a}(r,-i \nu), \quad \bar{\nu} \gg 1, \quad \text { for }|\arg t-\pi|<3 \pi / 4,
$$

where we are only neglecting exponentially-small corrections. Performing now a power series in $t / \bar{\nu}^{b}$ with $b \geq 1 / 6$ for $g_{a}(r, \pm i \nu) e^{ \pm i \pi \bar{\nu}} e^{\mp t^{2} / 2}$ (note that we must replace $e^{ \pm i \pi \bar{\nu}} \rightarrow e^{\mp i \pi \bar{\nu}}$ when $\arg (\bar{r}-1) \in(0, \pi] \rightarrow \arg (\bar{r}-1) \in(-\pi, 0)$, since this exponential comes from the $\ln (\bar{r}-1)$ in $\left.r_{*}=r_{*}(r)\right)$, we obtain

$$
\begin{array}{rlrl}
\forall s=0,1,2: & g_{a}(r, \pm i \nu) & \sim e^{\mp i \pi \bar{\nu}} e^{ \pm t^{2} / 2}\left[1+0 \frac{t^{0}}{\sqrt{\bar{\nu}}} \mp d_{1} \frac{t^{0}}{\bar{\nu}}+\ldots\right], \quad & \\
g_{a}(r, \pm i \nu) & \sim e^{ \pm i \pi \bar{\nu}} e^{ \pm t^{2} / 2}\left[1+0 \frac{t^{0}}{\sqrt{\bar{\nu}}} \mp d_{1} \frac{t^{0}}{\bar{\nu}}+\ldots\right], \quad & \text { if } \arg (\bar{r}-1) \in(0, \pi) \text { and } t \ll \bar{\nu}^{1 / 6}
\end{array}
$$

We will only require the coefficient $d_{1}$ for the case $s=1$, and in that case it is $d_{1}=-\lambda / 12$. We can then express the right-hand side of Eq. (3.25) as a linear combination of $\psi_{1}(t)$ and $\psi_{2}(t)$ for $\arg t=3 \pi / 4$, by using the asymptotics of Eqs. (3.19)-(3.21) at arg $t=\pi / 4$ together with the analytic continuation Eqs. (3.15)-(3.17). The result is

$$
\begin{aligned}
& s=0: \quad g_{\ell+}(r,-i \nu) e^{-i \pi \bar{\nu}} \sim-\frac{\sqrt{\pi \bar{\nu}}}{2}\left[3 i+\frac{(2-3 i) \alpha_{1}}{\sqrt{\bar{\nu}}}+O\left(\bar{\nu}^{-1}\right)\right] \psi_{1}(t)-\frac{2 \sqrt{\bar{\nu}}}{\sqrt{\pi}}\left[-1+\frac{\alpha_{1}}{\sqrt{\bar{\nu}}}+O\left(\bar{\nu}^{-1}\right)\right] \psi_{2}(t) \\
& s=1: \quad g_{\ell+}(r,-i \nu) e^{-i \pi \bar{\nu}} \sim \\
& \bar{\nu}\left[-1-\frac{\alpha_{1}}{\sqrt{\bar{\nu}}}+\frac{\lambda+12 i \alpha_{1}^{2}-6\left(\beta_{2}+\beta_{2}^{*}\right)+36 A}{12 \bar{\nu}}+O\left(\bar{\nu}^{-3 / 2}\right)\right] \psi_{1}(t)+ \\
& \overline{2}\left[1-\frac{\alpha_{1}}{\sqrt{\bar{\nu}}}+\frac{-\lambda-12 i \alpha_{1}^{2}+12 \alpha_{2}+6\left(\beta_{2}-\beta_{2}^{*}\right)}{12 \bar{\nu}}+O\left(\bar{\nu}^{-3 / 2}\right)\right] \psi_{2}(t) \\
& s=2: \quad g_{\ell+}(r,-i \nu) e^{-i \pi \bar{\nu}} \sim \frac{\sqrt{\pi}}{8}\left[3 i \bar{\nu}^{3 / 2}+(2-3 i) \alpha_{1} \bar{\nu}+O\left(\bar{\nu}^{1 / 2}\right)\right] \psi_{1}(t)+\frac{2}{\sqrt{\pi}}\left[-\frac{1}{\sqrt{\bar{\nu}}}+\frac{\alpha_{1}}{\bar{\nu}}+O\left(\bar{\nu}^{-3 / 2}\right)\right] \psi_{2}(t)
\end{aligned}
$$

which are valid $\forall \arg t \in[0,2 \pi)$, by analytic continuation. Using the asymptotics of Eqs.(3.19)-(3.21) for $\psi_{1,2}$ and rewriting the factors $e^{ \pm t^{2} / 2}$ in terms of the $g_{a}(r, \pm i \nu)$ using Eq. 3.25$)$, we find

$$
\begin{array}{cc}
s=0,2: & g_{\ell+}(r,-i \nu) \sim g_{a}(r,-i \nu)+(-1)^{1+s / 2} 2 e^{2 \pi i \bar{\nu}}\left[i+\frac{\alpha_{1}}{\sqrt{\bar{\nu}}}\right] g_{a}(r,+i \nu) \\
s=1: & g_{\ell+}(r,-i \nu) \sim\left[1+O\left(\bar{\nu}^{-2}\right)\right] g_{a}(r,-i \nu)+ \\
e^{2 \pi i \bar{\nu}}\left[-\frac{2 \alpha_{1}}{\sqrt{\bar{\nu}}}+\frac{2 i \alpha_{1}^{2}}{\bar{\nu}}+O\left(\bar{\nu}^{-3 / 2}\right)\right] g_{a}(r,+i \nu)
\end{array}
$$

The WKB expansion $g_{a}(r,+i \nu)$ is dominant over $g_{a}(r,-i \nu)$ in the region bounded by anti-Stokes lines which contains $r=r_{h}$, and so its coefficient can be trusted there.

In Fig 2 we plot the high-frequency asymptotics for $g_{\ell+}$ of Eq.(3.26) together with Eq.(3.1) (we only include a token plot, for $s=0$, since $g_{\ell+}$ does not appear explicitly in Eq.(2.9)). The figure shows that these asymptotics agree with the completely independent calculation of [29]. 


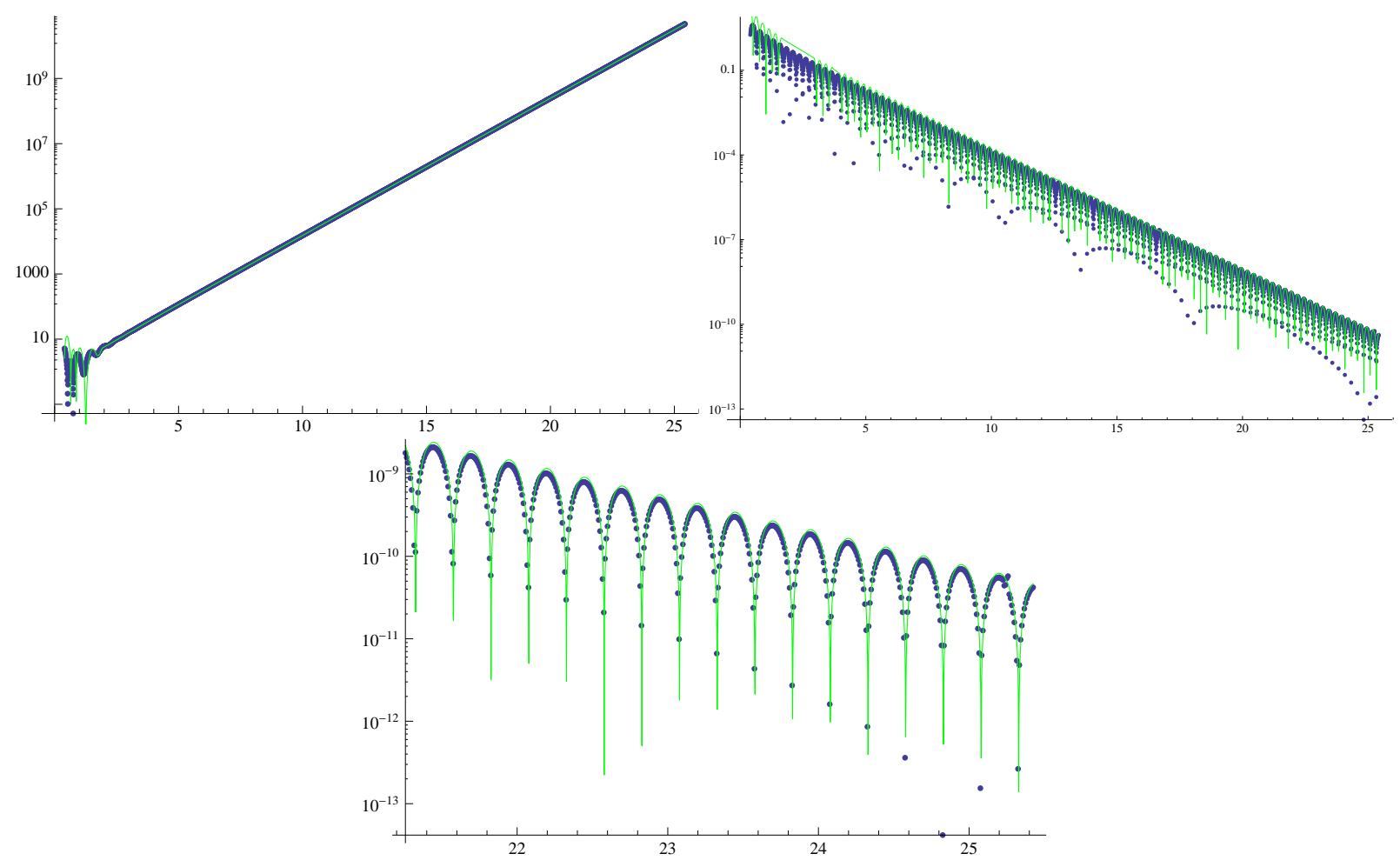

FIG. 2: Log-plots of $g_{\ell+}$ as a function of $\nu M$ for $s=0, \ell=1, r=2.8 M$. In blue: solution found using the method of [29]. In green: large- $\bar{\nu}$ asymptotics of Eq. 3.26). (a) $\left|\operatorname{Re}\left(g_{\ell+}\right)\right|$, (b) $\left|\operatorname{Im}\left(g_{\ell+}\right)\right|$, (c) 'zoomed-in' version of (b).

\section{BC 'strength' $q(\nu)$}

It follows from Eqs.(2.8) and (3.26) that $\Delta g_{\ell}(r,-i \nu)=2 i \operatorname{Im} g_{\ell}(r,-i \nu)=i q(\nu) g_{\ell}(r,+i \nu)$ with the following large- $\bar{\nu}$ asymptotics for the BC 'strength':

$$
\begin{array}{ll}
s=0,2: & q(\nu) \sim(-1)^{1+s / 2} 4\left[\cos (2 \pi \bar{\nu})+\frac{\alpha_{1}}{\sqrt{\bar{\nu}} \sin (2 \pi \bar{\nu})]+O\left(\bar{\nu}^{-1}\right)}\right. \\
s=1: & q(\nu) \sim 4 \alpha_{1}\left[-\frac{\sin (2 \pi \bar{\nu})}{\sqrt{\bar{\nu}}}+\frac{\alpha_{1} \cos (2 \pi \bar{\nu})}{\bar{\nu}}+O\left(\bar{\nu}^{-3 / 2}\right)\right]
\end{array}
$$

Alternatively, considering $q(\nu)$ in modulus-argument form we can rewrite Eq.(3.27) as

$$
\begin{aligned}
s & =0,2: & q(\nu) & \sim(-1)^{1+s / 2} 4\left[\cos \left(2 \pi \bar{\nu}-\frac{\alpha_{1}}{\sqrt{\bar{\nu}}}\right)+O\left(\bar{\nu}^{-1}\right)\right] \\
s & =1: & q(\nu) & \sim 4 \alpha_{1}\left[-\frac{1}{\sqrt{\bar{\nu}}} \sin \left(2 \pi \bar{\nu}-\frac{\alpha_{1}}{\sqrt{\bar{\nu}}}\right)+O\left(\bar{\nu}^{-3 / 2}\right)\right]
\end{aligned}
$$

since $g_{a}(r, \pm i \nu) \in \mathbb{R}$ and $g_{a}(r,+i \nu) \sim g_{\ell}(r,+i \nu)$, neglecting exponentially-small terms.

Figs 35 show that the large- $\bar{\nu}$ asymptotics of Eq. (3.27) match with a calculation of $q(\nu)$ using the independent method of [29] (where we will show more clearly that the curve corresponding to the latter method agrees with Figs.2 in [7] and [8). The asymptotics of Eq.(3.28) seem to do slightly better than those of Eq.(3.27) for $s=0$, slightly worse for $s=2$ while, for $s=1$, they seem to do better in the phase but worse in the amplitude. 


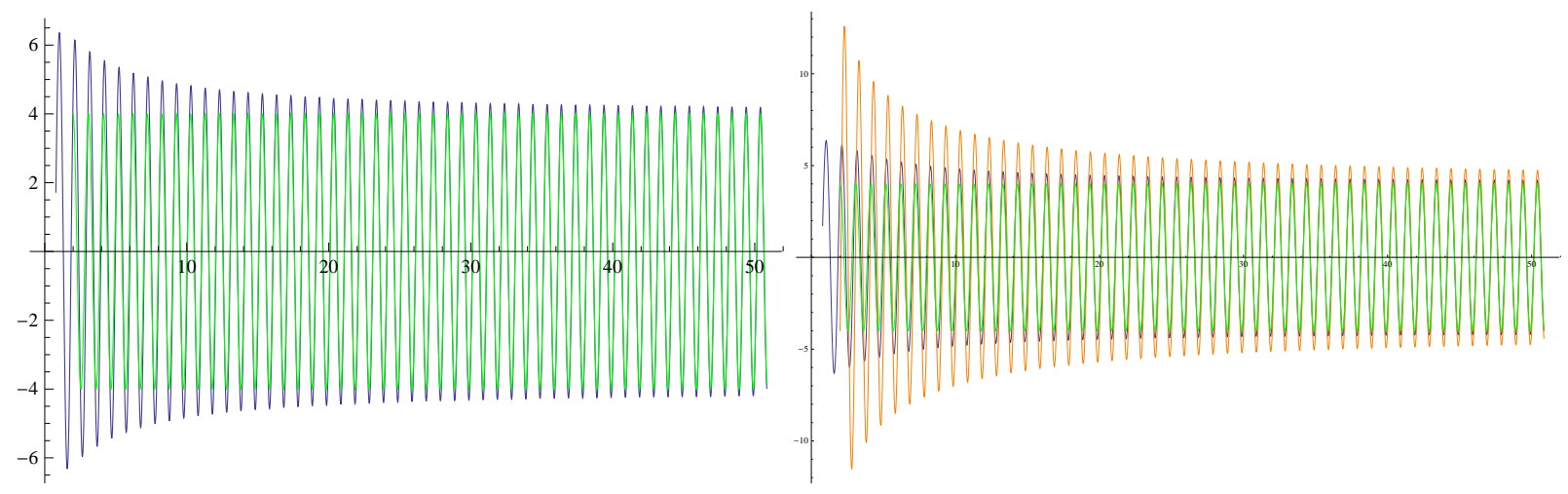

FIG. 3: BC 'strength' $q(\nu)$ of Eq. 2.8 as a function of $\bar{\nu}$ for $s=0, \ell=1$. (a) Green curve: large- $\bar{\nu}$ asymptotics of Eq. 3.28); blue curve: $q(\nu)$ calculated using the method of [29] via Eq.2.8 with the choice of value $r=5 M$. (b) Same as (a), where now we also include in orange the asymptotics of Eq. 3.27.

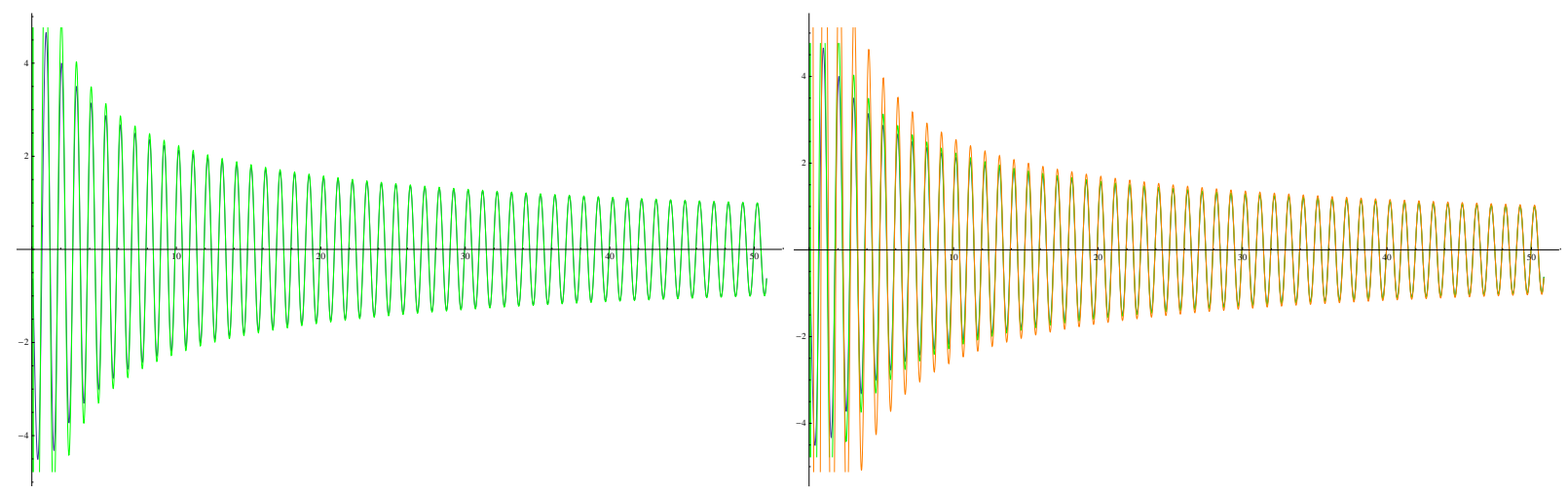

FIG. 4: $q(\nu)$ as a function of $\bar{\nu}$ for $s=1, \ell=1$. See caption in Fig 3 for a description of the curves.

\section{Radial solution $f_{\ell}(r,-i \nu)$}

From the boundary condition Eq.2.3 and the relationship $r_{*}=r_{*}(r)$, the following exact monodromy around the horizon follows:

$$
f_{\ell}\left(\left(r-r_{h}\right) e^{2 \pi i},-i \nu\right)=e^{-2 \pi i \bar{\nu}} f_{\ell}\left(r-r_{h},-i \nu\right)
$$

For $r$ near $r_{h}$ (but far away enough from it so that the WKB expansions $g_{a}$ are valid), it is $f_{\ell}(r,-i \nu) \sim e^{-\nu r_{*}} \sim g_{a}(r, i \nu)$ to leading order as $\bar{\nu} \gg 1$ and, since $g_{a}(r, i \nu)$ is there dominant over $g_{a}(r,-i \nu)$, we must have

$$
f_{\ell}(r,-i \nu) \sim g_{a}(r, i \nu)+c(\nu) g_{a}(r,-i \nu)
$$

for some function $c(\nu)$, where in the coefficient of $g_{a}(r, i \nu)$ we are potentially neglecting exponentially-small terms. Eq. (3.30 can be continued from the region of $r$ 'near' $r_{h}$ to the point $r_{*}=0$ and then anticlockwise along the anti-Stokes line up to $\arg (r)=\pi / 4$. From Eq. 3.25 , we then have that

$$
f_{\ell}(r,-i \nu) \sim e^{-i \pi \bar{\nu}} e^{t^{2} / 2}\left[1-\frac{d_{1}}{\bar{\nu}}+\cdots\right]+c(\nu) e^{i \pi \bar{\nu}} e^{-t^{2} / 2}\left[1+\frac{d_{1}}{\bar{\nu}}+\cdots\right]
$$




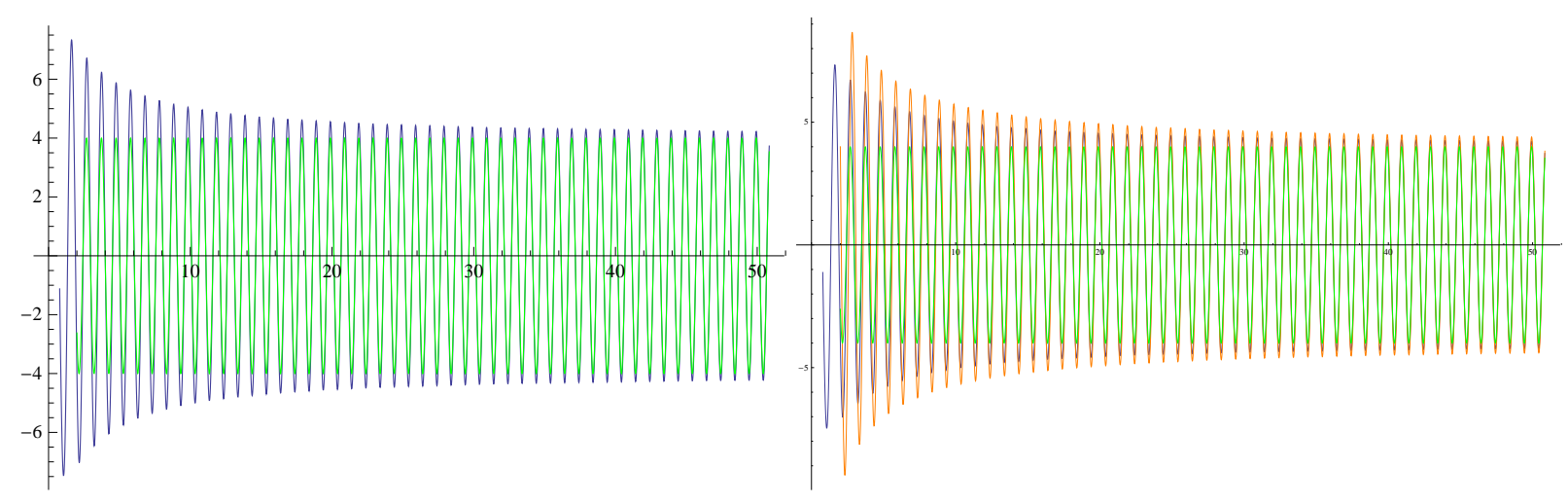

FIG. 5: $q(\nu)$ as a function of $\bar{\nu}$ for $s=2, \ell=2$. See caption in Fig 3 for a description of the curves. Cf. Fig.2 7 ] (also Fig.2 [8]).

for $1 / \sqrt{\bar{\nu}} \ll|\bar{r}| \ll 1$ with $\arg (r)=\pi / 4$. From Eqs. 3.19-3.21 we find that

$$
\begin{aligned}
& s=0: \quad f_{\ell}(r,-i \nu) \sim \frac{\sqrt{\pi \bar{\nu}}}{2}\left\{e^{-i \pi \bar{\nu}}\left[1+\frac{(i-2) \alpha_{1}}{\sqrt{\bar{\nu}}}\right]-c(\nu) e^{i \pi \bar{\nu}}\left[i+\frac{(i+2) \alpha_{1}}{\sqrt{\bar{\nu}}}\right]\right\} \psi_{1}+ \\
& \frac{2 \sqrt{\bar{\nu}}}{\sqrt{\pi}}\left\{e^{-i \pi \bar{\nu}}\left[i-\frac{\alpha_{1}}{\sqrt{\bar{\nu}}}\right]-c(\nu) e^{i \pi \bar{\nu}}\left[1+\frac{\alpha_{1}}{\sqrt{\bar{\nu}}}\right]\right\} \psi_{2}, \\
& s=1: \quad f_{\ell}(r,-i \nu) \sim \\
& \frac{\bar{\nu}}{2}\left\{e^{-i \pi \bar{\nu}}\left[1-\frac{i \alpha_{1}}{\sqrt{\bar{\nu}}}+\frac{i \alpha_{1}^{2}-\operatorname{Re}\left(\beta_{2}\right)+A}{\bar{\nu}}\right]\left(1-\frac{d_{1}}{\bar{\nu}}\right)-c(\nu) e^{i \pi \bar{\nu}}\left[1-\frac{\alpha_{1}}{\sqrt{\bar{\nu}}}+\frac{i \alpha_{1}^{2}+\operatorname{Re}\left(\beta_{2}\right)-A}{\bar{\nu}}\right]\left(1+\frac{d_{1}}{\bar{\nu}}\right)\right\} \psi_{1}+ \\
& \left\{e^{-i \pi \bar{\nu}}\left[1+\frac{i \alpha_{1}}{\sqrt{\bar{\nu}}}+\frac{i \alpha_{1}^{2}-\alpha_{2}-i \operatorname{Im}\left(\beta_{2}\right)}{\bar{\nu}}\right]\left(1-\frac{d_{1}}{\bar{\nu}}\right)+c(\nu) e^{i \pi \bar{\nu}}\left[1+\frac{\alpha_{1}}{\sqrt{\bar{\nu}}}+\frac{i \alpha_{1}^{2}+\alpha_{2}-i \operatorname{Im}\left(\beta_{2}\right)}{\bar{\nu}}\right]\left(1+\frac{d_{1}}{\bar{\nu}}\right)\right\} \psi_{2}, \\
& s=2: \quad f_{\ell}(r,-i \nu) \sim \frac{\sqrt{\pi \bar{\nu}^{3}}}{8}\left\{e^{-i \pi \bar{\nu}}\left[1+\frac{(i-2) \alpha_{1}}{\sqrt{\bar{\nu}}}\right]+c(\nu) e^{i \pi \bar{\nu}}\left[i+\frac{(i+2) \alpha_{1}}{\sqrt{\bar{\nu}}}\right]\right\} \psi_{1}+ \\
& \frac{2}{\sqrt{\pi \bar{\nu}}}\left\{e^{-i \pi \bar{\nu}}\left[i-\frac{\alpha_{1}}{\sqrt{\bar{\nu}}}\right]+c(\nu) e^{i \pi \bar{\nu}}\left[1+\frac{\alpha_{1}}{\sqrt{\bar{\nu}}}\right]\right\} \psi_{2}
\end{aligned}
$$

This expression has been obtained with all functions $f_{\ell}, \psi_{1}, \psi_{2}$ evaluated for $\arg (r)=\pi / 4$, but it is valid $\forall \arg (r)$. In particular, we can express $\psi_{1}$ and $\psi_{2}$ on $\arg (r)=-\pi / 4$ in terms of $e^{ \pm t^{2} / 2}$ by using $\operatorname{Eqs} .3 .19-3.21$ on $\arg (r)=\pi / 4$ together with the analytic continuation Eqs.3.15)-3.17. We can match this expression to a linear combination of $g_{a}( \pm i \nu)$ via Eq. 3.1. This linear combination can then be continued anticlockwise along the anti-Stokes line all the way back to $r_{*}=0$, thus yielding the asymptotic monodromy:

$$
\begin{aligned}
s=0: & f_{\ell}\left(\left(r-r_{h}\right) e^{2 \pi i},-i \nu\right)=e^{-2 \pi i \bar{\nu}} g_{a}\left(r-r_{h}, i \nu\right)+\left[-2 i\left(1-\frac{\alpha_{1}}{\sqrt{\bar{\nu}}}\right)+c(\nu) e^{2 \pi i \bar{\nu}}\right] g_{a}\left(r-r_{h},-i \nu\right), \\
s=1: \quad & f_{\ell}\left(\left(r-r_{h}\right) e^{2 \pi i},-i \nu\right)= \\
& \left\{e^{-2 \pi i \bar{\nu}}\left[1+O\left(\bar{\nu}^{-3 / 2}\right)\right]+c(\nu) O\left(\bar{\nu}^{-3 / 2}\right)\right\} g_{a}\left(r-r_{h}, i \nu\right)+ \\
& \left\{\frac{2 i \alpha_{1}}{\sqrt{\bar{\nu}}}-\frac{2 i \alpha_{1}^{2}}{\bar{\nu}}+O\left(\bar{\nu}^{-3 / 2}\right)+c(\nu) e^{2 \pi i \bar{\nu}}\left[1+O\left(\bar{\nu}^{-3 / 2}\right)\right]\right\} g_{a}\left(r-r_{h},-i \nu\right), \\
s=2: \quad & f_{\ell}\left(\left(r-r_{h}\right) e^{2 \pi i},-i \nu\right)=e^{-2 \pi i \bar{\nu}} g_{a}\left(r-r_{h}, i \nu\right)+\left[2 i\left(1-\frac{\alpha_{1}}{\sqrt{\bar{\nu}}}\right)+c(\nu) e^{2 \pi i \bar{\nu}}\right] g_{a}\left(r-r_{h},-i \nu\right),
\end{aligned}
$$


Finally, comparing with the exact monodromy Eq. 3.29) and using Eq. (3.30), we obtain

$$
\begin{aligned}
& s=0,2: \quad c(\nu) \sim(-1)^{s / 2} \frac{\left(1-\alpha_{1} / \sqrt{\bar{\nu}}\right)}{\sin (2 \pi \bar{\nu})}, \\
& s=1: \quad c(\nu) \sim\left[\frac{2 i \alpha_{1}}{\sqrt{\bar{\nu}}}-\frac{2 i \alpha_{1}^{2}}{\bar{\nu}}+O\left(\bar{\nu}^{-3 / 2}\right)\right]\left[-2 i \sin (2 \pi \bar{\nu})-e^{2 \pi i \bar{\nu}} O\left(\bar{\nu}^{-3 / 2}\right)\right]^{-1}
\end{aligned}
$$

as well as, for $s=1$ and from the coefficient of $g_{a}\left(r-r_{h}, i \nu\right)$,

$$
e^{-2 \pi i \bar{\nu}}=e^{-2 \pi i \bar{\nu}}\left[1+O\left(\bar{\nu}^{-3 / 2}\right)\right]+c(\nu) O\left(\bar{\nu}^{-3 / 2}\right)
$$

Note that the terms $O\left(\bar{\nu}^{-3 / 2}\right)$ in Eq. 3.35 may actually decrease faster than $\bar{\nu}^{-3 / 2}$ and they do not necessarily have to decrease both at the same rate. In particular, this implies that, e.g., $c(\nu)$ may increase/decrease with $\bar{\nu}$ as long as the $O\left(\bar{\nu}^{-3 / 2}\right)$ multiplying it makes up for it by decreasing/increasing faster than the other $O\left(\bar{\nu}^{-3 / 2}\right)$.

In Fig. 6 we plot $\hat{f}_{\ell}=-\sin (2 \pi \bar{\nu}) f_{\ell}(r,-i \nu)$ as a function of $M \nu$ : it shows that the large- $\bar{\nu}$ asymptotics of Eq. 3.30 (together with (3.34) and (3.1) ) overlap with a calculation of $\hat{f}_{\ell}$ using the so-called Jaffé series [29, 34].

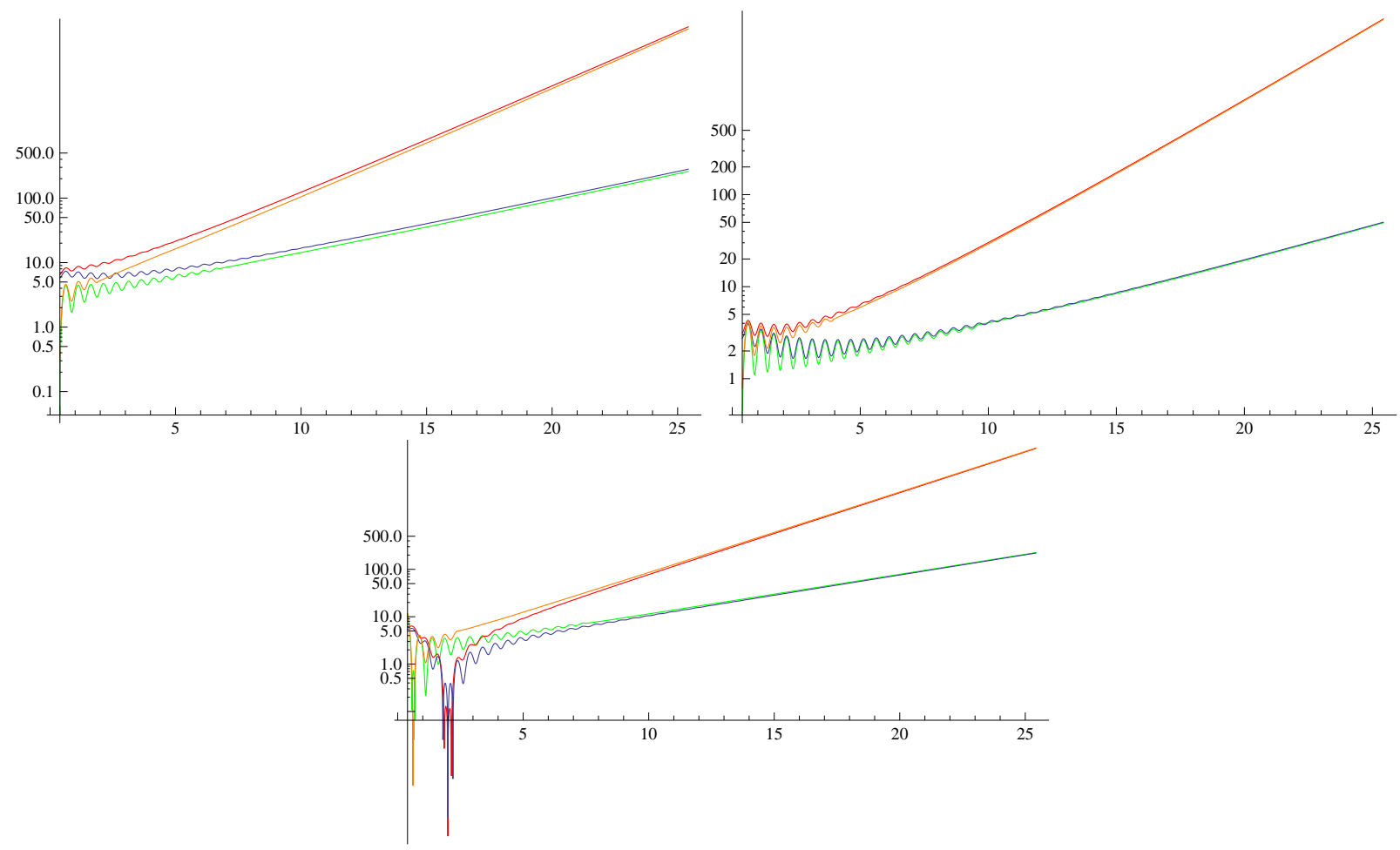

FIG. 6: Log-plot of $\left|\hat{f}_{\ell}\right|$ as a function of $\nu M$. The green and orange curves are the large- $\bar{\nu}$ asymptotics of Eq. 3.30 (together with (3.34) and (3.1) for $r_{*}=0.2 M$ and $r_{*}=0.4 M$ respectively. The blue and red curves are respectively calculated using the Jaffé series 29, 34 for $r_{*}=0.2 M$ and $r_{*}=0.4 M$. (a) For $s=0, \ell=1$. (b) For $s=1, \ell=1$. (c) For $s=2, \ell=2$; note that both curves for the Jaffé series give a zero value for $\hat{f}_{\ell}$ at $\bar{\nu}=\bar{\nu}_{A S}$ because of the definition of $\hat{f}_{\ell}$ and the fact that, for $s=2, \bar{\nu}_{A S}$ is not a pole. 


\section{E. Wronskian}

Using Eq. 3.26) for $g_{\ell+}$ and Eqs.(3.30), (3.34) and (3.1) for $f_{\ell}$, we find the following large- $\bar{\nu}$ asymptotics for the Wronskian on the 4 th quadrant of the complex- $\omega$ plane infinitesimally close to the NIA:

$$
\begin{array}{cl}
s=0,2: & -2 \nu A_{\ell, \omega+}^{i n}=W_{+}(-i \nu) \sim \frac{W\left[g_{a}(-i \nu), g_{a}(i \nu)\right]}{\sin (2 \pi \bar{\nu})}\left[\sin (2 \pi \bar{\nu})+2 i e^{2 \pi i \bar{\nu}}\left(1-\frac{(1+i) \alpha_{1}}{\sqrt{\bar{\nu}}}\right)\right], \\
s=1: & -2 \nu A_{\ell, \omega+}^{i n}=W_{+}(-i \nu) \sim W\left[g_{a}(-i \nu), g_{a}(i \nu)\right]\left\{1+O\left(\bar{\nu}^{-3 / 2}\right)+\left[\frac{2 \alpha_{1}}{\sqrt{\bar{\nu}}}-\frac{2 i \alpha_{1}^{2}}{\bar{\nu}}+O\left(\bar{\nu}^{-3 / 2}\right)\right] c(\bar{\nu}) e^{2 \pi i \bar{\nu}}\right\}
\end{array}
$$

To leading order for $s=0$ and $s=2$ it yields $W_{+}(-i \nu) \sim 2 \nu[1-2 i \cot (2 \pi \bar{\nu})]$. This agrees with Eq.2.17 in [12]:

$$
\left.A_{\ell, \omega-}^{i n} \sim \lim _{\epsilon \rightarrow 0^{+}}\left(\frac{e^{-4 \pi \bar{\omega}}-1}{e^{-4 \pi \bar{\omega}}+[1+2 \cos (\pi s)]}\right)^{-1}\right|_{\bar{\omega}=-\epsilon-\bar{\nu} i}, \quad \bar{\nu} \gg 1,
$$

on the 3rd quadrant infinitesimally close to the NIA, after using the symmetry Eq. 2.6.

Similarly to the 'trick' used to go from Eq.3.27) to Eq.3.28, we can rewrite Eq. 3.36 as

$$
\begin{aligned}
& s=0,2: \quad W_{+}(-i \nu) \sim \frac{W\left[g_{a}(-i \nu), g_{a}(i \nu)\right]}{\sin (2 \pi \bar{\nu})}\left[\sin (2 \pi \bar{\nu})+2 i e^{2 \pi i \bar{\nu}-\frac{(1+i) \alpha_{1}}{\sqrt{\bar{\nu}}}}\right], \\
& s=1: \quad W_{+}(-i \nu) \sim W\left[g_{a}(-i \nu), g_{a}(i \nu)\right]\left\{1+O\left(\bar{\nu}^{-3 / 2}\right)-\frac{2 \alpha_{1}^{2}}{\bar{\nu} \sin (2 \pi \bar{\nu})} e^{2 \pi i \bar{\nu}-\frac{(1+i) \alpha_{1}}{\sqrt{\bar{\nu}}}}\right\}
\end{aligned}
$$

where we have used Eq. (3.34) in the $s=1$ case.

In Figs.7 9 we plot $\hat{W}_{+}(-i \nu)=W\left[g_{\ell+}(r,-i \nu), \hat{f}_{\ell}(r,-i \nu)\right]$ as a function of $M \nu$ and we show that the large- $\bar{\nu}$ asymptotics of Eq.3.36 agree with the calculation using the method in [29.

\section{F. Green function modes}

We can finally give an asymptotic expression for large- $\bar{\nu}$ for the BC discontinuity of the 'retarded' Green function modes. From Eq. 2.9 and the leading orders of Eqs. 3.36), (3.30) and (3.27), we find

$$
\begin{aligned}
& \Delta G_{\ell}\left(r, r^{\prime} ;-i \nu\right) \sim \frac{(-1)^{s / 2} 2 i}{\nu} \frac{\cos (2 \pi \bar{\nu})}{\left[1+3 \cos ^{2}(2 \pi \bar{\nu})\right]}\left[(-1)^{s / 2} e^{\nu r_{*}}+\sin (2 \pi \bar{\nu}) e^{-\nu r_{*}}\right]\left[(-1)^{s / 2} e^{\nu r_{*}^{\prime}}+\sin (2 \pi \bar{\nu}) e^{-\nu r_{*}^{\prime}}\right], \quad s=0,2 \\
& \Delta G_{\ell}\left(r, r^{\prime} ;-i \nu\right) \sim \frac{-\sqrt{\pi} i \ell(\ell+1) \sin (2 \pi \bar{\nu}) r_{h}}{\bar{\nu}^{3 / 2}}\left[\frac{\sqrt{\pi} \ell(\ell+1)}{2 \bar{\nu}^{1 / 2} \sin (2 \pi \bar{\nu})} e^{\nu r_{*}}+e^{-\nu r_{*}}\right]\left[\frac{\sqrt{\pi} \ell(\ell+1)}{2 \bar{\nu}^{1 / 2} \sin (2 \pi \bar{\nu})} e^{\nu r_{*}^{\prime}}+e^{-\nu r_{*}^{\prime}}\right]
\end{aligned}
$$

This shows the convergence of the $\nu$-integral in the upper limit of integration in Eq. (2.7) when $\Delta t>\left|r_{*}\right|+\left|r_{*}^{\prime}\right|$. The divergence in the $\mathrm{BC}$ when $\Delta t<\left|r_{*}\right|+\left|r_{*}^{\prime}\right|$ was to be expected, since the QNM series also seems to diverge at these 'very early' times [2, 6], and one would expect the divergences in the different contributions to the Green function to cancel each other out (see, e.g., 38] for the case of a radial potential which does not lead to a BC, and where the divergences of the QNM and high-frequency arc contributions at these 'very early' times cancel out). In Figs 10 11 we plot the large- $\bar{\nu}$ asymptotics of $\Delta G_{\ell}\left(r, r^{\prime} ; \omega\right)$ together with these modes calculated with the method in [29]. 


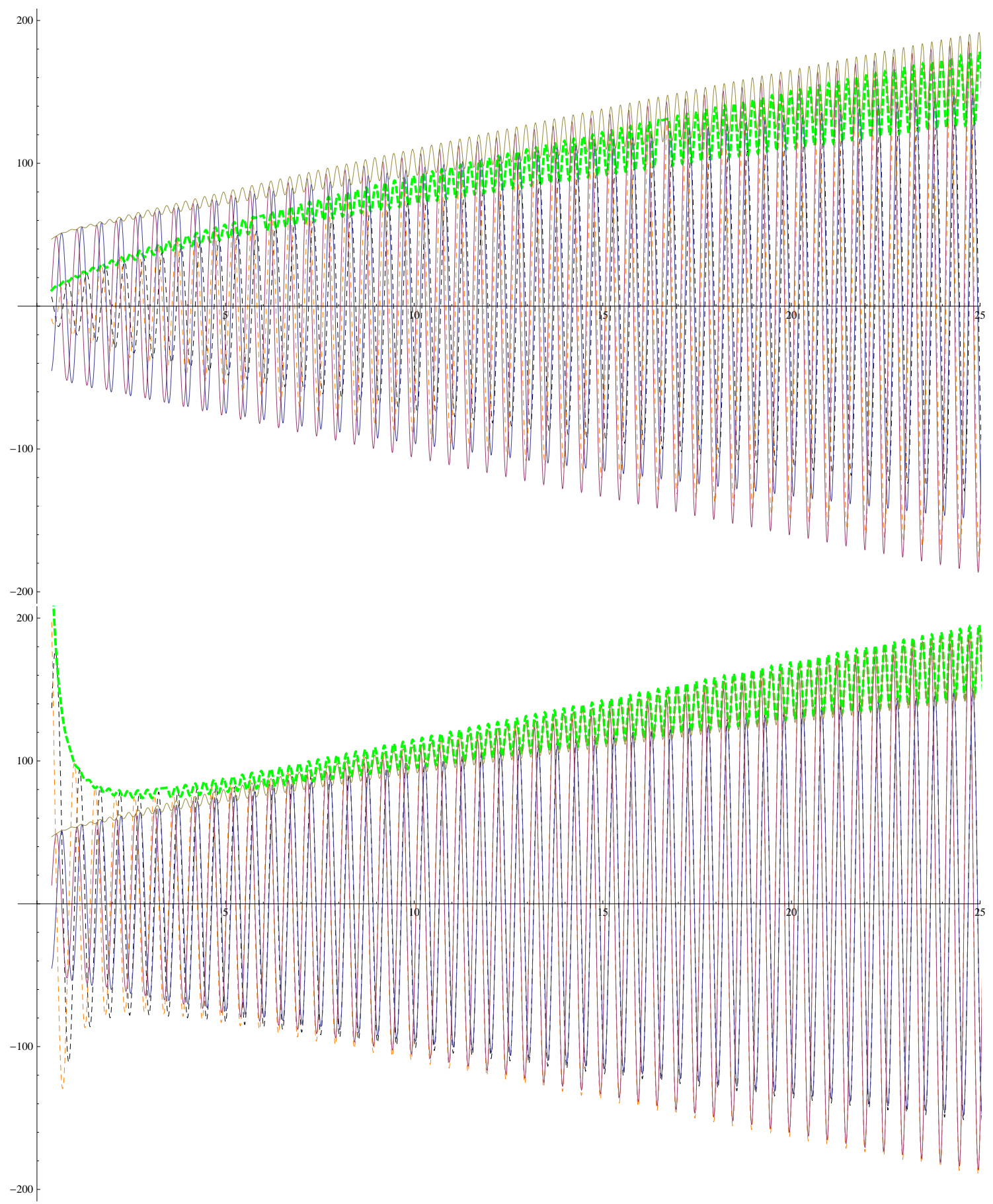

FIG. 7: Real part, imaginary part and absolute value of $\hat{W}_{+}$as functions of $\nu M$. The asymptotic expressions in Eq. 3.36 . are plotted as dashed black, orange and green curves, corresponding to the real part, imaginary part and absolute value of $\hat{W}_{+}$, respectively. The calculation using the method in 29 (for this, the value $r=2.8 M$ has been used to calculate the radial solutions $g_{\ell+}$ and $\hat{f}_{\ell}$ ) is plotted as continuous blue, red and brown curves, corresponding to the real part, imaginary part and absolute value of $\hat{W}_{+}$, respectively. This plot is for the values $s=0, \ell=1$. (a) Asymptotic expressions using Eq. (3.36). (b) Asymptotic expressions using Eq. (3.38). The odd non-oscillatory intervals in the dashed green curves are just a visual artifact of the computational software program that we used for plotting the curves. 


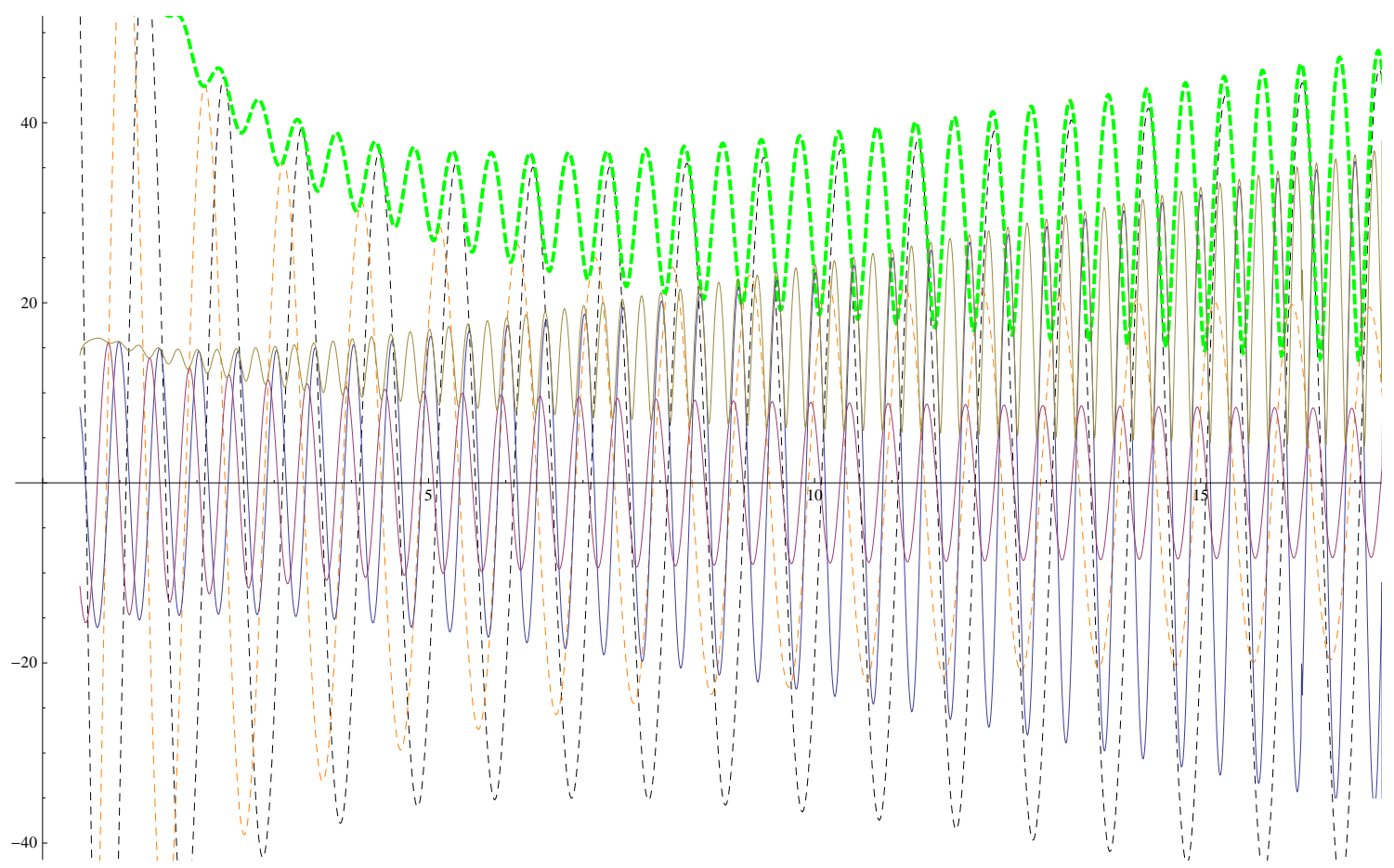

FIG. 8: Real part, imaginary part and absolute value of $\hat{W}_{+}$as functions of $\nu M$. This plot is for the values $s=1, \ell=1$. See caption in Fig]7(a) for a description of the curves. Note that, in this case, the method in [29] (corresponding to the blue curve) cannot reach higher values of $\nu M$.

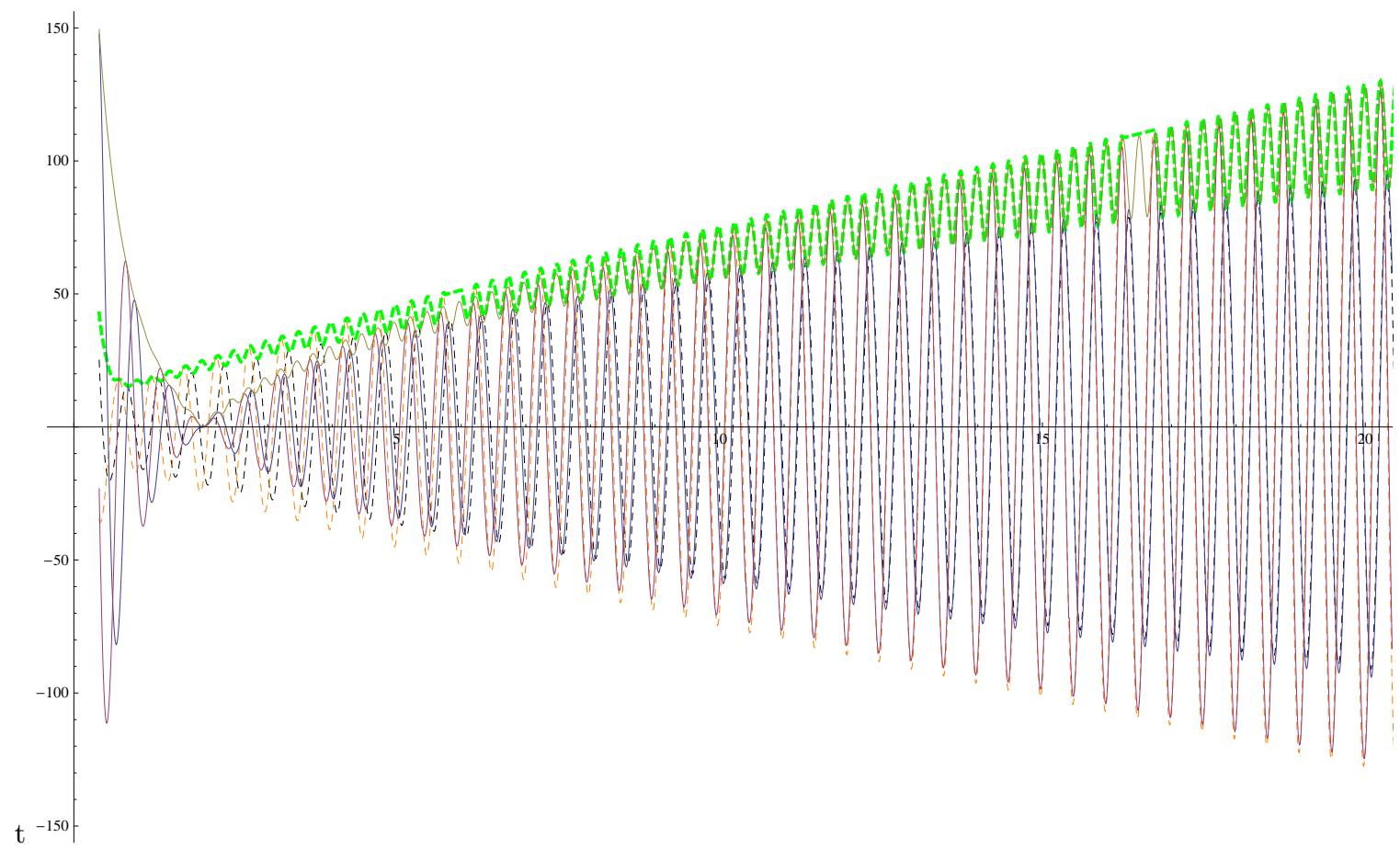

FIG. 9: Real part, imaginary part and absolute value of $\hat{W}_{+}$as functions of $\nu M$. This plot is for the values $s=2, \ell=2$. See caption in Fig 7(a) for a description of the curves. Note that the blue and black curves are mostly overlapping, and so are the orange and red curves. The curves obtained with the method in 29] have a zero at $\nu=\nu_{A S}$ due to a corresponding zero in $\hat{f}_{\ell}$. 


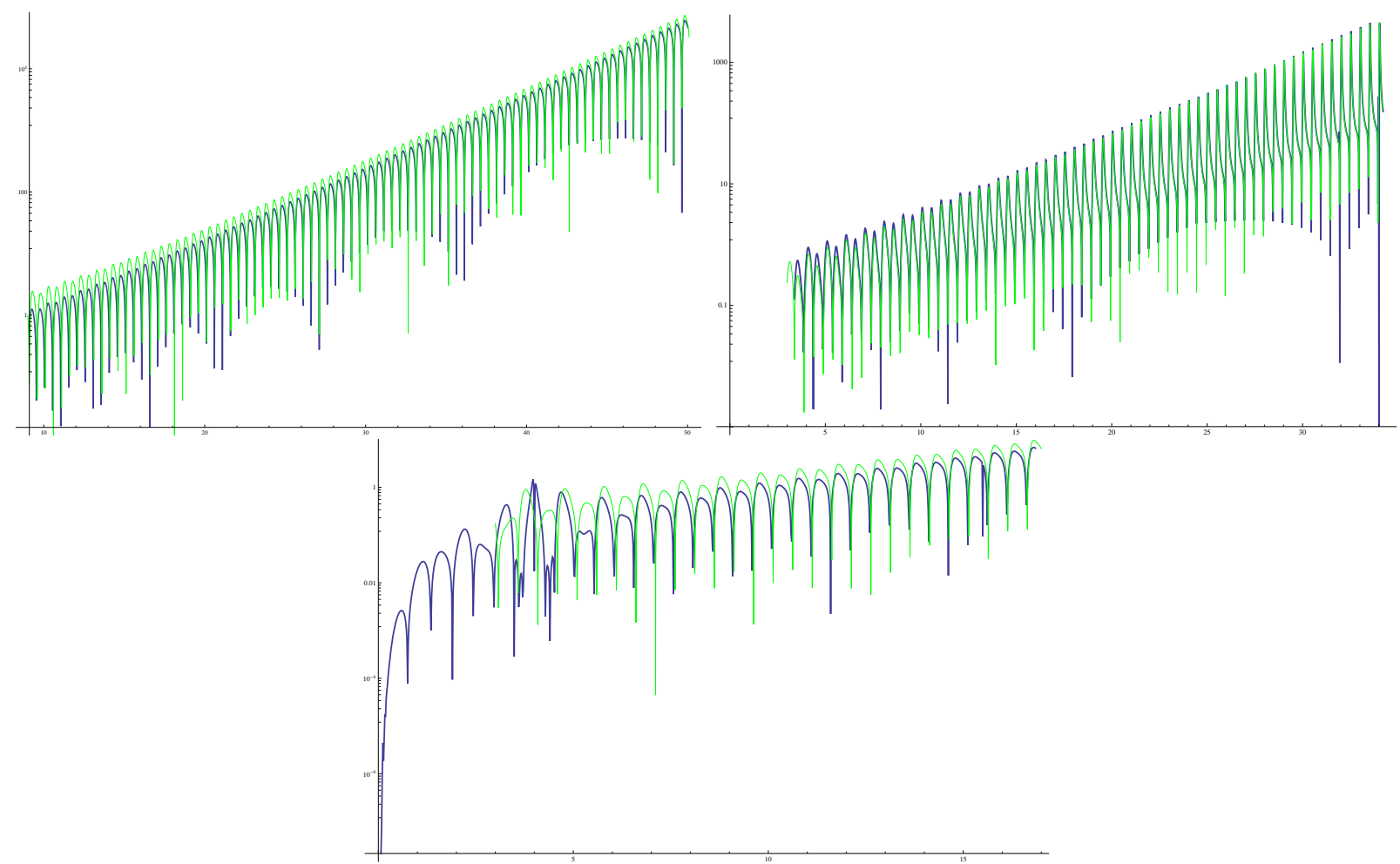

FIG. 10: BC Green function modes $\Delta G_{\ell}\left(r\left(r_{*}=0.4\right), r^{\prime}\left(r_{*}=0.2\right) ;-i \nu\right)$ of Eq. 2.9 as a function of $\bar{\nu}$. The green curves correspond to the large- $\bar{\nu}$ asymptotics of $\Delta G_{\ell}$ using Eqs. (3.27), (3.36) and (3.30) (so not just the leading-order Eq.(3.39)). The blue curves have been obtained with the method in 29 (for which we have used $\hat{f}_{\ell}$ instead of $f_{\ell}$ everywhere in Eq. 2.9 and we have chosen to calculate the radial functions at $r=5 M$ and at $r=2.8 M$ for the calculation of, respectively, $q(\nu)$ and the Wronskian). (a) For $s=0$ and $\ell=1$. (b) For $s=1$ and $\ell=1$. (c) For $s=2$ and $\ell=2$ (the 'particular' behaviour around $\bar{\nu}=4$ is due to this value being that of the algebraically-special frequency).

\section{HIGHLY-DAMPED QNMS}

QNMs are poles of the 'retarded' Green function in the lower frequency plane. By requiring that $W_{+}=-2 \nu A_{\ell, \omega+}^{i n}$ in Eq.3.36 be zero, we obtain the highly-damped QNM frequencies

$$
\begin{array}{ll}
s=0: & \bar{\omega}_{l n} \sim \frac{\ln 3}{4 \pi}-\left(\frac{n}{2}+\frac{1}{4}\right) i+\frac{\sqrt{2} \Gamma^{4}(1 / 4)}{144 \pi^{5 / 2}}(1+i) \frac{3 \lambda+1}{\sqrt{n}}+O\left(\frac{1}{n}\right), \quad n \rightarrow \infty \\
s=2: & \bar{\omega}_{l n} \sim \frac{\ln 3}{4 \pi}-\left(\frac{n}{2}+\frac{1}{4}\right) i+\frac{\sqrt{2} \Gamma^{4}(1 / 4)}{144 \pi^{5 / 2}}(1+i) \frac{\lambda-1}{\sqrt{n}}+O\left(\frac{1}{n}\right),
\end{array}
$$

where $n$ is the so-called overtone index. These expressions agree with 21] (for $s=2$ we are just including the result in [10] for completeness).

For $s=1$, 21, 23] show that the $O(1)$ and $O\left(n^{-1 / 2}\right)$ terms in the QNM frequencies are zero as $n \rightarrow \infty$. In 24] they find numerical indications that for $s=1$ the highly-damped quasinormal modes go like

$$
\bar{\omega}_{l n} \sim \frac{i n}{2}+\frac{a_{3} \lambda^{3}+a_{2} \lambda^{2}+a_{1} \lambda}{n^{3 / 2}}, \quad n \rightarrow \infty
$$

with undetermined polynomial coefficients $a_{1}, a_{2}$ and $a_{3}$. For $s=1$, from Eq. (3.36) we would find the QNM condition to be

$$
1+O\left(\bar{\nu}^{-3 / 2}\right)+\left[\frac{2 \alpha_{1}}{\sqrt{\bar{\nu}}}-\frac{2 i \alpha_{1}^{2}}{\bar{\nu}}+O\left(\bar{\nu}^{-3 / 2}\right)\right] c(\bar{\nu}) e^{2 \pi i \bar{\nu}}=0
$$




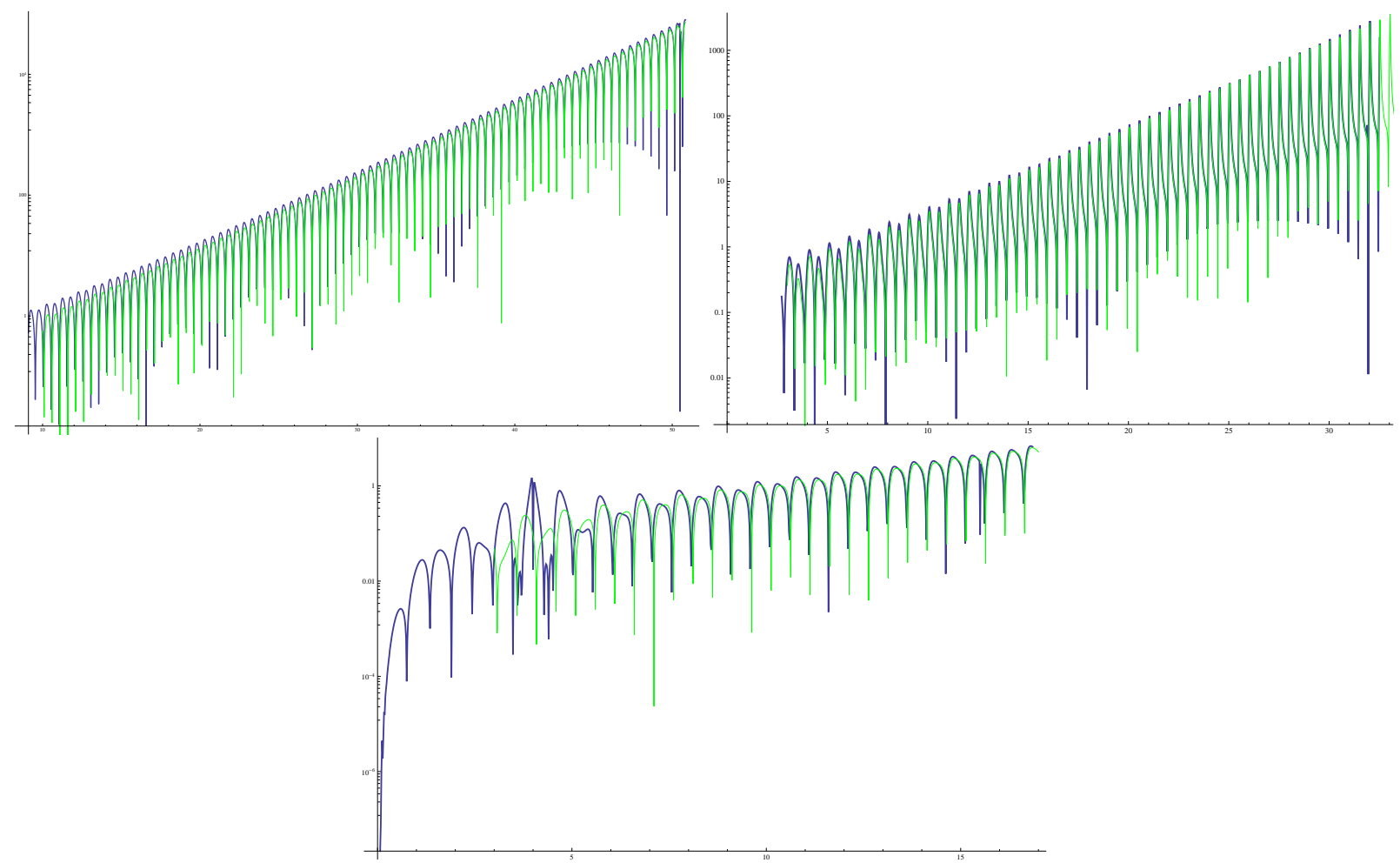

FIG. 11: Similar to Figs 10 but using Eq. 3.38 instead of Eq. 3.36. It shows a slight improvement with respect to Figs 10 .

Let us try first with the asymptotic expression $2 \pi \bar{\nu} \sim n \pi+a / n^{3 / 2}$, for some undetermined coefficient $a$. We then obtain, from Eq. 3.34, that $c(\nu)=O(n)=O(\bar{\nu})$, but in Eq. 4.3 we obtain a leading order $O(\sqrt{n})$, which cannot be cancelled. Trying then with $2 \pi \bar{\nu} \sim n \pi+a+\ldots$ or $2 \pi \bar{\nu} \sim n \pi+a \sqrt{n}+\ldots$ yields $c(\nu)=O\left(n^{-1 / 2}\right)$ but the 1 in Eq. 4.3 cannot be cancelled. Trying next with $2 \pi \bar{\nu} \sim n \pi+a / \sqrt{n}+\ldots$ yields $c(\nu)=O(1)$, but then the 1 in Eq. 4.3 . cannot be cancelled either. Finally, let us try with $2 \pi \bar{\nu} \sim n \pi+a / n+b / n^{3 / 2}$, and then we obtain, from Eq.(3.34), that $c(\nu)=O\left(n^{1 / 2}\right)=O\left(\bar{\nu}^{1 / 2}\right)$ and from Eq.4.3 it follows that: $a=4 \alpha_{1}^{2}$ and $b=-4 \sqrt{2}(1+i) \alpha_{1}^{3}$. Therefore, we have that the highly-damped electromagnetic QNM frequencies are given by

$$
\bar{\omega}_{l n}=-\frac{i n}{2}-\frac{i \lambda^{2}}{2 n}+\frac{\pi^{1 / 2}(1-i) \lambda^{3}}{2^{3 / 2} n^{3 / 2}}+O\left(\frac{1}{n^{2}}\right), \quad s=1
$$

This form agrees with the form of the numerics of Eq.4.2) (considering that only the leading order in the imaginary part is shown) and Fig, 12 shows that it also agrees with the numerical data in [39].

\section{HIGH-FREQUENCY BC RESPONSE TO A PERTURBATION}

In this section we consider a perturbation to a Schwarzschild black hole and we investigate the high-frequency BC contribution of the $\ell$-mode to the response to such perturbation. If the perturbation is given by some initial conditions $u_{\ell}^{i c}\left(r_{*}^{\prime}\right) \equiv u_{\ell}\left(r_{*}^{\prime}, t^{\prime}=0\right)$ and $\dot{u}_{\ell}^{i c}\left(r_{*}^{\prime}\right) \equiv \partial_{t} u_{\ell}\left(r_{*}^{\prime}, t^{\prime}=0\right)$, then the full $\ell$-mode response is given by

$$
u_{\ell}\left(r_{*}, t\right)=\int_{-\infty}^{\infty} d r_{*}^{\prime}\left[G_{\ell}^{r e t}\left(r, r^{\prime} ; t\right) \dot{u}_{\ell}^{i c}\left(r_{*}^{\prime}\right)+u_{\ell}^{i c}\left(r_{*}^{\prime}\right) \partial_{t} G_{\ell}^{r e t}\left(r, r^{\prime} ; t\right)\right]
$$

while the $\mathrm{BC}$ contribution to the response is given by

$$
u_{\ell}^{B C}\left(r_{*}, t\right) \equiv \int_{-\infty}^{\infty} d r_{*}^{\prime}\left[G_{\ell}^{B C}\left(r, r^{\prime} ; t\right) \dot{u}_{\ell}^{i c}\left(r_{*}^{\prime}\right)+u_{\ell}^{i c}\left(r_{*}^{\prime}\right) \partial_{t} G_{\ell}^{B C}\left(r, r^{\prime} ; t\right)\right]
$$



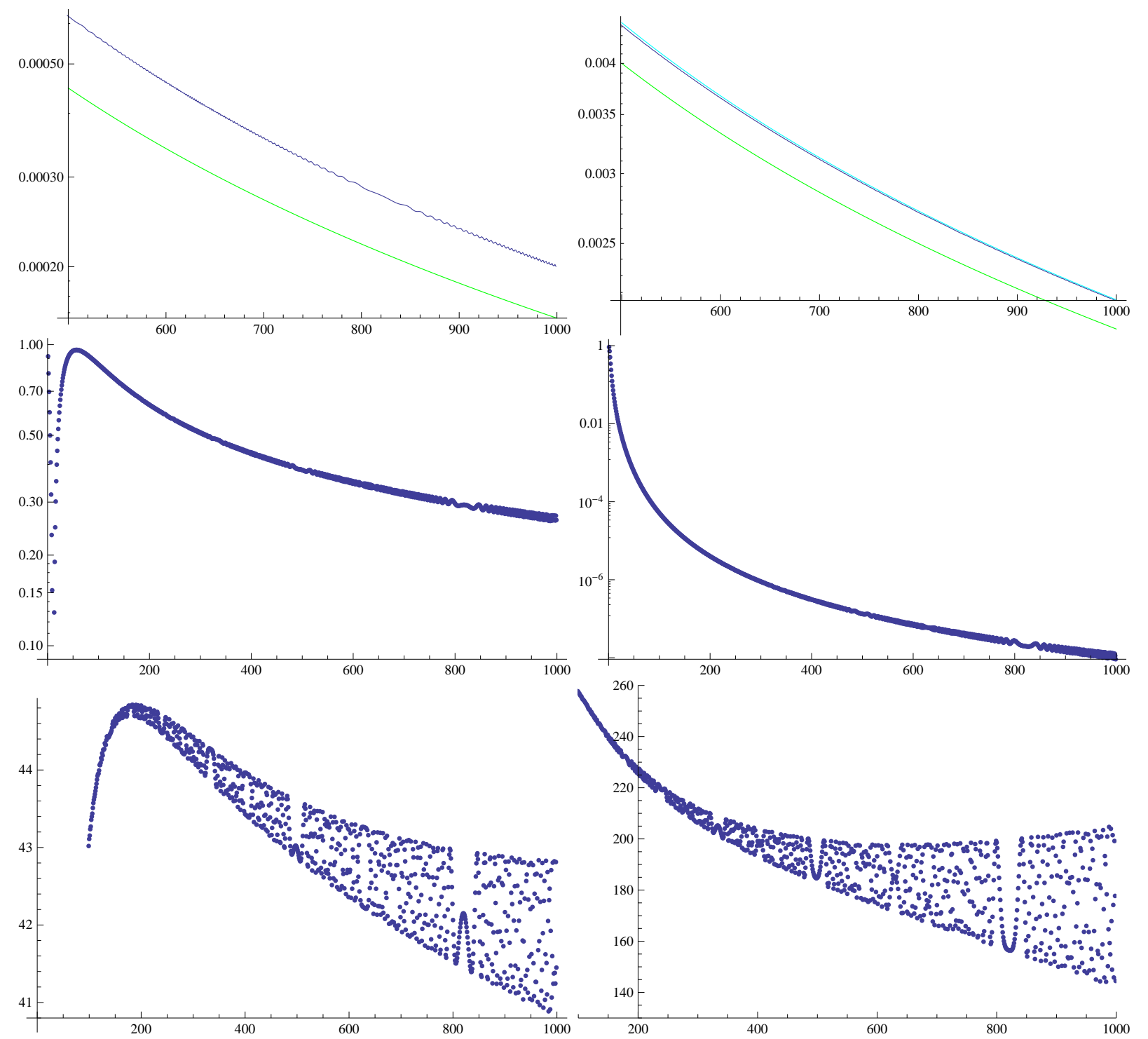

FIG. 12: Comparison of the QNM frequencies $\bar{\omega}_{l n}$ given by the asymptotic expression Eq. 4.4), which we will denote by $\bar{\omega}_{l n}^{a}$, and the numerical data in 39] (after complex-conjugating it in order to account for the different definition of the frequency), which we will denote by $\bar{\omega}_{l n}^{n}$. For $s=1$ and $\ell=1$. Figs.(a)-(d) are log-plots. Horizontal axis: overtone index $n$. (a) In blue: $\left|\operatorname{Re}\left(\bar{\omega}_{l n}^{n}\right)\right|$; in green: $\left|\operatorname{Re}\left(\bar{\omega}_{l n}^{a}\right)\right|$. (b) In (dark) blue: $\left|\operatorname{Im}\left(\bar{\omega}_{l n}^{n}\right)+\frac{n}{2}\right|$; in green: the leading order for $\left|\operatorname{Im}\left(\bar{\omega}_{l n}^{a}\right)+\frac{n}{2}\right|$, i.e., $\frac{\lambda^{2}}{2 n} ;$ in light-blue: $\left|\operatorname{Im}\left(\bar{\omega}_{l n}^{a}\right)+\frac{n}{2}\right|$. (c): 'Relative error' || $\operatorname{Re}\left(\bar{\omega}_{l n}^{n}\right) / \operatorname{Re}\left(\bar{\omega}_{l n}^{a}\right)|-1|$. (d): Same as (c) but for the imaginary part. (e) Plot of $n^{2} \cdot \operatorname{Re}\left(\bar{\omega}_{l n}^{n}-\bar{\omega}_{l n}^{a}\right)$. (f) Plot of $n^{5 / 2} \cdot \operatorname{Im}\left(\bar{\omega}_{l n}^{n}-\bar{\omega}_{l n}^{a}\right)$. Figs.(e) and (f) show, respectively, that the difference between the asymptotic Eq. 4.4 and the numerical data in 39] is only at order $n^{-2}$ for the real part and $n^{-5 / 2}$ for the imaginary part.

We will consider the case $\dot{u}_{\ell}^{i c}\left(r_{*}^{\prime}\right)=\partial_{r_{*}^{\prime}} u_{\ell}^{i c}\left(r_{*}^{\prime}\right)$, corresponding to an initial wave-packet moving towards the left. In addition, for convenience, we take a normalization given by $\int_{-\infty}^{\infty} d r_{*}^{\prime}\left|u_{\ell}^{i c}\left(r_{*}^{\prime}\right)\right|=1$. Let us define $F_{\ell}(r, \omega) \equiv$ $f_{\ell}(r, \omega) e^{-i \omega r_{*}}$, and by analogy with $\hat{f}_{\ell}$ define $\hat{F}_{\ell}(r,-i \nu)=-\sin (2 \pi \bar{\nu}) F(r,-i \nu)$. Introducing Eq. 2.9 into Eq. 5.2 . with these initial conditions, we obtain:

$$
\begin{aligned}
& u_{\ell}^{B C}\left(r_{*}, \Delta t\right)=\int_{0}^{\infty} d \nu I(r, \nu), \quad I(r, \nu) \equiv-e^{-\nu\left(\Delta t-r_{*}\right)} \frac{\nu q(\nu) \hat{F}_{\ell}(r,-i \nu)}{\pi\left|\hat{W}_{ \pm}\right|^{2}} \hat{C}_{\ell}(\nu) \\
& C_{\ell}(\nu) \equiv \int_{-\infty}^{+\infty} d r_{*} F_{\ell}(r,-i \nu) e^{\nu r_{*}^{\prime}}\left[-\nu+\partial_{r_{*}}\right] u_{\ell}^{i c}\left(r_{*}\right), \quad \hat{C}_{\ell}(\nu) \equiv-\sin (2 \pi \bar{\nu}) C_{\ell}(\nu) .
\end{aligned}
$$


We have defined the function $C_{\ell}(\nu)$ so that, when evaluated at the quasinormal mode frequencies, it corresponds to the 'excitation coefficients' $\mathcal{C}_{\ell n}$ defined below for the QNM series.

We will now investigate the behaviour for large- $\bar{\nu}$. From Eq. 3.30,

$$
F_{\ell}(r,-i \nu) \sim c(\nu)+e^{-2 \nu r_{*}} \sim \begin{cases}\frac{(-1)^{s / 2}}{\sin (2 \pi \bar{\nu})}+e^{-2 \nu r_{*}}, & s=0,2 \\ \frac{\lambda \sqrt{\pi}}{2 \sqrt{\bar{\nu}} \sin (2 \pi \bar{\nu})}+e^{-2 \nu r_{*}}, & s=1\end{cases}
$$

for $\bar{\nu} \rightarrow \infty$. From Eqs. 3.27) and (3.36, we have that as $\bar{\nu} \rightarrow \infty$

$$
\begin{aligned}
& s=0,2: \quad \frac{-2 \nu q(\nu)}{\left|\hat{W}_{ \pm}\right|^{2}} \sim \frac{(-1)^{s / 2} 2 \cos (2 \pi \bar{\nu})}{\nu\left[1+3 \cos ^{2}(2 \pi \bar{\nu})\right]} \\
& s=1: \quad \frac{-2 \nu q(\nu)}{\left|\hat{W}_{ \pm}\right|^{2}} \sim \frac{-\lambda \sqrt{\pi} r_{h}}{\bar{\nu}^{3 / 2} \sin (2 \pi \bar{\nu})\left|1-\frac{\lambda^{2} \pi e^{2 \pi i \bar{\nu}}}{2 \bar{\nu} \sin (2 \pi \bar{\nu})}\right|^{2}}
\end{aligned}
$$

In order to study the large- $\nu$ asymptotics for $C_{\ell}(\nu)$, we first re-express it as

$$
C_{\ell}(\nu)=-2 \nu c(\nu) \int_{-\infty}^{\infty} d r_{*} e^{\nu r_{*}} u_{\ell}^{i c}\left(r_{*}\right)
$$

after integration by parts, where we have used Eq.(5.4) and we have assumed that the initial conditions are such that the boundary terms are zero, i.e., that $F_{\ell}\left(r_{*}\right) e^{\nu r_{*}} u_{\ell}^{\imath c}\left(r_{*}\right) \rightarrow 0$ as $r_{*} \rightarrow \pm \infty$.

It is clear that if the initial conditions have compact support, with $u_{\ell}^{i c}\left(r_{*}\right)=0$ for all $r_{*}>R_{*}$, then $\left|C_{\ell}(\nu)\right| \leq$ $2|c(\nu)| \nu e^{\nu R_{*}}$. Together with the leading-order Eq. (5.5), this determines that the integrand $I(r, \nu)$ in Eq. (5.3) goes, at most, like $e^{\nu\left(-\Delta t+\left|r_{*}\right|+R_{*}\right)}$ as $\bar{\nu} \rightarrow \infty$ (ignoring powers of $\bar{\nu}$ ). Therefore, if the initial data is of compact support, the $\nu$-integral in the $\mathrm{BC}$ contribution to the perturbation response will converge after a certain time: for $\Delta t>\left|r_{*}\right|+R_{*}$.

Let us now consider the case of non-compact initial conditions, specifically the case of a Gaussian distribution centered at $r_{*}=x_{0}$ and moving towards the black hole, which has frequently been used in the literature:

$$
u_{\ell}^{i c}\left(r_{*}\right)=\frac{1}{\sqrt{2 \pi} \sigma} \exp \left(\frac{-\left(r_{*}-x_{0}\right)^{2}}{2 \sigma^{2}}\right), \quad \dot{u}_{\ell}^{i c}\left(r_{*}\right)=-\frac{\left(r_{*}-x_{0}\right)}{\sigma^{2}} u_{\ell}^{i c}\left(r_{*}\right)
$$

for some $\sigma \in \mathbb{R}$. Note that Eq. (5.7) is the case of Fig.2 of Leaver [2] with $\sigma=2^{-1 / 2}(2 M)^{2}$ (apart from a different overall constant factor). The high-frequency asymptotics of $C_{\ell}(\nu)$ for the initial conditions (5.7) are then given by:

$$
\begin{aligned}
s=0,2: & \hat{C}_{\ell}(\nu) \sim(-1)^{s / 2} \frac{2 \bar{\nu}}{r_{h}} e^{\sigma^{2} \nu^{2} / 2} e^{\nu x_{0}}, \quad \bar{\nu} \rightarrow \infty, \\
s=1: & \hat{C}_{\ell}(\nu) \sim \frac{\lambda \sqrt{\bar{\nu} \pi}}{r_{h}} e^{\sigma^{2} \nu^{2} / 2} e^{\nu x_{0}} .
\end{aligned}
$$

In Fig 13 we plot $\left|\hat{C}_{\ell}(\nu)\right|$ as a function of $M \nu$. The leading-orders in Eqs. (5.5) and (5.8) together yield that the integrand $I(r, \nu)$ is of the order of $e^{\sigma^{2} \nu^{2} / 2-\nu\left(\Delta t-\left|r_{*}\right|-x_{0}\right)}$ as $\bar{\nu} \rightarrow \infty$ (ignoring powers of $\bar{\nu}$ ). This asymptotic behaviour implies that the $\nu$-integral in Eq.(5.3) will not converge for any given values of $\Delta t$ and $r_{*}$. See Fig 14 for a plot of $\left|I(r, \nu) e^{\nu T}\right|$. Leaver's Sec.III.A 2 considers the particular initial perturbation Eq. (5.7) that we have used here. Eqs.54-56 2], however, only investigate the 'late-time response', i.e., they are obtained via Eqs.38-44 [2], which are obtained for $\bar{r} \gg 1$ and $\bar{\nu} \ll 1$. This is probably the reason why the large- $\bar{\nu}$ divergence observed here went unnoticed in [2]. This large- $\bar{\nu}$ divergence for any $\Delta t$ and $r_{*}$ for Gaussian initial data is in contrast with the convergence for $\Delta t>\left|r_{*}\right|+R_{*}$ in the case of initial data with compact support that we have seen above.

We expect a similar behaviour for the highly-damped QNM's in the overtone $n$-sum for the QNM contribution to the perturbation response:

$$
u_{\ell}^{Q N M}\left(r_{*}, t\right)=2 \sum_{n=0}^{\infty} u_{\ell, n}^{Q N M}\left(r_{*}, t\right), \quad u_{\ell, n}^{Q N M}\left(r_{*}, t\right) \equiv \operatorname{Re}\left(\frac{\mathcal{B}_{\ell n}}{\left(A_{\ell, \omega_{l n}}^{\text {out }}\right)^{2}} \mathcal{C}_{\ell n} F_{\ell}\left(r_{*}, \omega_{l n}\right) e^{-i \omega_{l n}\left(\Delta t-r_{*}\right)}\right)
$$


where $\mathcal{C}_{\ell n} \equiv C_{\ell}\left(\nu_{l n}\right)$, the QNM 'excitation factors' are defined by $\mathcal{B}_{\ell n} \equiv A_{\ell, \omega_{l n}}^{\text {out }} /\left(\omega_{l n} \alpha_{\ell n}\right)$, and $\alpha_{\ell n}$ is defined via $A_{\ell, \omega}^{i n} \sim\left(\omega-\omega_{l n}\right) \alpha_{\ell n}$ as $\omega \rightarrow \omega_{l n}$. The $n$-sum in Eq. 5.9$)$ is over all QNMs in the fourth quadrant of the complex- $\omega$ plane. We note that the radial function $g_{\ell}$ does not appear in Eq. 5.9 because we may replace it by $f_{\ell} / A_{\ell, \omega}^{\text {out }}$ at a QNM frequency: the two quantities are equal when $\omega=\omega_{l n}$, as follows from the fact that $A_{\ell, \omega_{l n}}^{\text {in }}=0$ and from the boundary conditions 2.3 and 2.4 .

Comparing Eq. 5.9 with the BC contribution Eq. 5.3 , we can say that: $i \omega_{\ell n}, 2 \operatorname{Re} \sum_{n=0}^{\infty}, \frac{\mathcal{B}_{\ell n}}{\left(A_{\ell, \omega_{l n}}^{\text {out }}\right)^{2}}, \mathcal{C}_{\ell n}$ in the QNM contribution 'play the rôle' of, respectively: $\nu, \int_{0}^{\infty} d \nu, \frac{-\nu q(\nu)}{\pi\left|W_{ \pm}\right|^{2}}, C_{\ell}(\nu)$ in the BC contribution. This is particularly true in the high-damping limit, $n \rightarrow \infty$.

Let us find the large- $n$ asymptotics of $\mathcal{B}_{\ell n}$. By comparing Eqs. 2.4 and 3.30 for $r \rightarrow \infty$ we have that $A_{\ell, \omega}^{\text {out }} \sim c(\nu)$ as $\bar{\nu} \rightarrow \infty$, which is valid since $A_{\ell, \omega}^{\text {out }}$ is the coefficient of the dominant solution for $r_{*}>0$. From Eq. 3.34) it then follows that, to leading order as $\bar{\nu} \rightarrow \infty$,

$$
\begin{aligned}
s=0,2: \quad A_{\ell, \omega}^{\text {out }} & \sim \frac{(-1)^{s / 2}}{\sin (2 \pi \bar{\nu})}, \\
s=1: \quad A_{\ell, \omega}^{\text {out }} & \sim \frac{\sqrt{\pi} \lambda}{2 \sqrt{\bar{\nu}} \sin (2 \pi \bar{\nu})},
\end{aligned}
$$

in the 4 th quadrant of the complex- $\omega$ plane. In order to compare with 12 , let us note that our definition of tortoise coordinate $r_{*}$ equals that in Eq.B.1 [12] plus the constant ' $i \pi$ '. Therefore, our coefficients $A_{\ell, \omega}^{\text {in }}$ and $A_{\ell, \omega}^{\text {out }}$ correspond, respectively, to $1 / T_{N}$ and $e^{-2 \pi i \bar{\nu}} R_{N} / T_{N}$, where $R_{N}$ and $T_{N}$ are the reflection and transmission coefficients defined by Neitzke via Eq.2.10 [12]. Eq.(5.10) for $s=0$ and 2 then agrees with Eqs.2.17 and 2.18 [12], after also taking into account a different sign in the definition of $\omega$ and converting expression (5.10) from the 4th quadrant into the 3rd quadrant by using the symmetries (2.5). Note that the different constant of integration ' $i \pi^{\prime}$ ' in $r_{*}=r_{*}(r)$ is probably the reason why $A_{\ell, \omega}^{\text {out }}$ in Eq. 5.10 for $s=0$ and 2 differs by a factor $e^{-2 \pi i \bar{\nu}}$ from Eq.A.2 [40]. In the Appendix A we calculate the relationship between the coefficients $A_{\ell, \omega}^{\text {out }}$ and $A_{\ell, \omega}^{i n}$ following a different contour in the complex- $\omega$ plane, thus providing a check of Eq. 5.10. We can now calculate that as $n \rightarrow \infty$

$$
\begin{array}{r}
s=0,2: \quad \alpha_{\ell n} \sim 3 \pi r_{h}, \quad \mathcal{B}_{\ell n} \sim \frac{(-1)^{n+s / 2} i}{\sqrt{3} \pi n}, \\
s=1: \quad \alpha_{\ell n} \sim \frac{2 i r_{h} n}{\lambda^{2}}, \quad \mathcal{B}_{\ell n} \sim \frac{(-1)^{n} \lambda}{\sqrt{2 \pi} n^{3 / 2}},
\end{array}
$$

where we have used Eq. 3.36) to obtain $\alpha_{\ell n}$ and Eqs.4.1) and 4.4 for the highly-damped QNM frequencies. The behaviour $\mathcal{B}_{\ell n}=O\left(n^{-1}\right)$ for $s=0$ seems to roughly agree with Fig.2 6] (although those results are not really meant to be valid for large- $n)$. To the best of our knowledge, the expressions in (5.10) and $(5.11)$ for the $s=1$ case are given here for the first time in the literature.

The asymptotics as $n \rightarrow \infty$ for $F_{\ell}\left(r_{*}, \omega_{l n}\right)$ and $\mathcal{C}_{\ell n}$ are those of $F_{\ell}\left(r_{*},-i \nu\right)$ and $C_{\ell}(\nu)$ in Eqs. (5.4) and (5.6), respectively, with the replacement $\nu \rightarrow i \omega_{\ell n}$. Combining the asymptotics of Eqs.(5.4), (5.10) and (5.11) we find that, for $n \rightarrow \infty$,

$$
\begin{aligned}
& s=0,2: \quad u_{\ell, n}^{Q N M}\left(r_{*}, t\right) \sim \frac{(-1)^{n+s / 2} 4}{3 \sqrt{3} \pi n} \operatorname{Re}\left[i \mathcal{C}_{\ell n}\left(c\left(\nu_{l n}\right) e^{-\nu_{l n}\left(\Delta t-r_{*}\right)}+e^{-\nu_{l n}\left(\Delta t+r_{*}\right)}\right)\right] \\
& s=1: \quad u_{\ell, n}^{Q N M}\left(r_{*}, t\right) \sim \frac{(-1)^{n} \lambda^{3} \sqrt{2 \pi}}{n^{5 / 2}} \operatorname{Re}\left[\mathcal{C}_{\ell n}\left(c\left(\nu_{l n}\right) e^{-\nu_{l n}\left(\Delta t-r_{*}\right)}+e^{-\nu_{l n}\left(\Delta t+r_{*}\right)}\right)\right]
\end{aligned}
$$

where a right-moving and a left-moving wave in the radial direction can be seen, both exponentially-damped with time. In particular, if the initial data is of compact support vanishing for $r_{*}>R_{*}$, then, for large- $n,\left|\mathcal{C}_{\ell n}\right|<\left|c\left(\nu_{l n}\right)\right| n e^{n R_{*} / 2}$ and it then follows from Eq. (5.12) that the QNM $n$-sum in Eq.(5.9) will be convergent for $\Delta t>\left|r_{*}\right|+R_{*}$, just like the corresponding BC contribution.

Let us now look at the case of Gaussian initial data, Eq.5.7); from Eq.5.8 we find as $n \rightarrow \infty$

$$
\begin{array}{ll}
s=0,2: & \mathcal{C}_{\ell n} \sim \frac{(-1)^{s / 2+1+n} \sqrt{3} n}{2 r_{h}} e^{\bar{\sigma}^{2}\left[N^{2}+\frac{\ln 3}{\pi} i N-\frac{\ln ^{2} 3}{2 \pi^{2}}\right] / 8} e^{N \bar{x}_{0} / 2+i \bar{x}_{0} \ln 3 /(4 \pi)}, \quad N \equiv n+1 / 2 \\
s=1: & \mathcal{C}_{\ell n} \sim \frac{(-1)^{n+1} n^{3 / 2}}{\sqrt{2 \pi} \lambda r_{h}} e^{\bar{\sigma}^{2} n^{2} / 8} e^{n \bar{x}_{0} / 2}
\end{array}
$$


The divergence of $\mathcal{C}_{\ell n}$ for large- $n$ is not cancelled out by any other quantity in Eq. (5.12), so that the QNM $n$-sum for the non-compact initial data (5.7) is not convergent, just like the $\nu$-integral in the corresponding BC contribution. Specifically, from Eqs. 5.12 and (5.13),

$$
\begin{aligned}
& s=0,2: \quad u_{\ell, n}^{Q N M}\left(r_{*}, t\right) \sim-\frac{2}{3 \pi r_{h}} e^{\bar{\sigma}^{2}\left(N^{2}-\frac{\ln ^{2} 3}{4 \pi^{2}}\right) / 8+N x_{0} / 2} \times \\
& {\left[\frac{(-1)^{s / 2+n} \sqrt{3}}{2} e^{-N\left(\Delta \bar{t}-\bar{r}_{*}\right) / 2} \sin \left(\frac{\ln 3}{4 \pi}\left(\frac{\bar{\sigma}^{2} N}{2}-\Delta \bar{t}+\bar{r}_{*}+\bar{x}_{0}\right)\right)+e^{-N\left(\Delta \bar{t}^{2}+\bar{r}_{*}\right) / 2} \sin \left(\frac{\ln 3}{4 \pi}\left(\frac{\bar{\sigma}^{2} N}{2}-\Delta \bar{t}-\bar{r}_{*}+\bar{x}_{0}\right)\right)\right]} \\
& s=1: \quad u_{\ell, n}^{Q N M}\left(r_{*}, t\right) \sim-\frac{\lambda^{2}}{r_{h} n} e^{\bar{\sigma}^{2} n^{2} / 8+n \bar{x}_{0} / 2}\left[\frac{(-1)^{n} \sqrt{n}}{\sqrt{2 \pi} \lambda} e^{-n\left(\Delta \bar{t}-\bar{r}_{*}\right) / 2}+e^{-n\left(\Delta \bar{t}+\bar{r}_{*}\right) / 2}\right]
\end{aligned}
$$

A naïve attempt at the calculation of the corresponding large-frequency divergence in the BC contribution indicates that it does not cancel out the large- $n$ divergence in the QNM contribution in the case of Gaussian initial data, although it is hard to be definitive given the numerous simultaneous asymptotic limits and integrals involved. On the other hand, the full perturbation response Eq.(5.1) is known to be well defined, therefore, we expect the high-frequency arc contribution together with the BC and QNM contributions to be regular, with the divergences in the different contributions cancelling each other out. We also expect a similar cancellation between the high-frequency divergences from the BC, QNM and high-frequency arc contributions in the case of compact initial data for $\Delta t<\left|r_{*}\right|+x_{0}$.

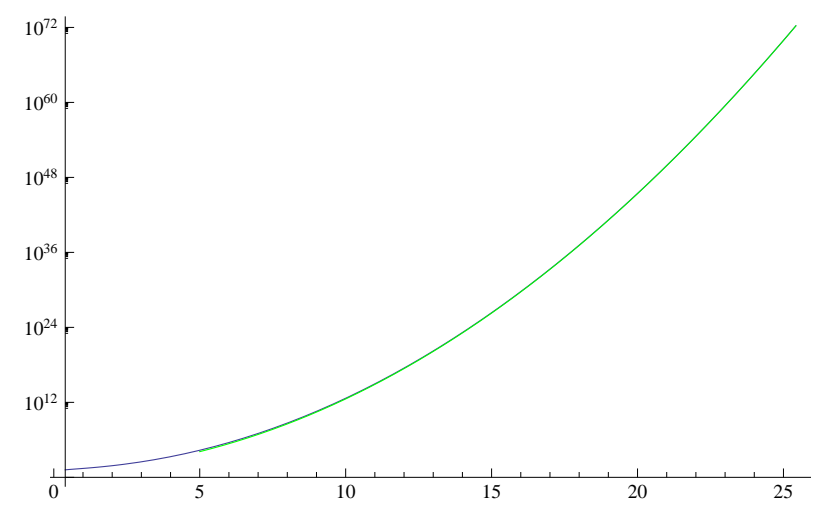

FIG. 13: Log-plot of $\left|\hat{C}_{\ell}(\nu)\right|$ in Eq. 5.3 as a function of $\nu M$ for $s=0$ and $\ell=1$. The blue curve is obtained with the method in 29]. The overlapping curve in green is obtained using the large- $\nu$ asymptotics of Eq. 3.30 for $\hat{F}_{\ell}$ in Eq. 5.3. The coefficient $\hat{C}_{\ell}(\nu), \forall \nu$, for both curves has been obtained by integrating using the built-in function NIntegrate from $r=r_{h}$ up to $\infty$ in the computational software program Mathematica.

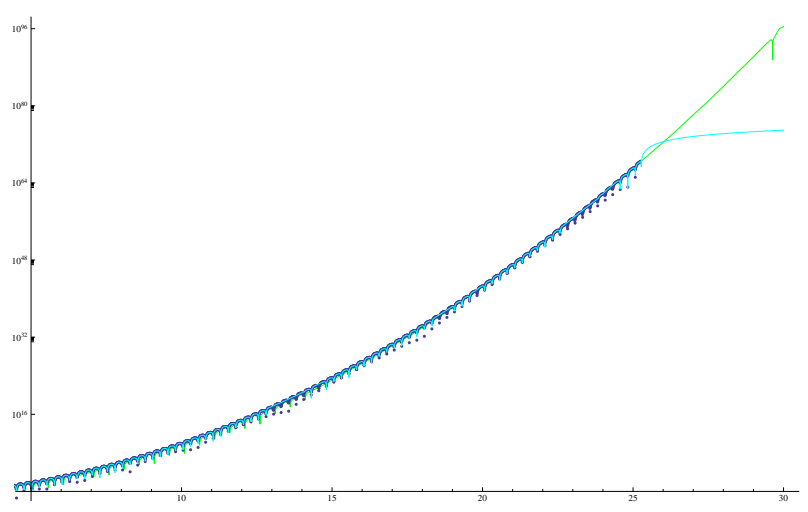

FIG. 14: Log-plot as a function of $\nu M$ of $|I(r=10 M, \nu)|$ in Eq. 5.3 but without including the factor $e^{-\nu T}$. For $s=0$ and $\ell=1$. The darker blue curve is obtained with the method in [29] and the light-blue curve is the interpolation of this data. The (partly overlapping) green curve is obtained with the large- $\nu$ asymptotics: Eq. 3.27) for $q(\nu)$, Eq. 3.30 for $\hat{F}_{\ell}$, Eq. 3.36 for the Wronskian and $C_{\ell}(\nu)$ as in the green curve in Fig 13 


\section{CONCLUSIONS}

The branch cut contribution to the Green function in the Schwarzschild spacetime has scarcely been investigated in the literature, except in the small-frequency regime. The challenging technical difficulties for the calculation away from that asymptotic regime is probably a reason for it. However, in order to have an understanding of the full response of the black hole to an initial perturbation or, in general, to know the Green function globally, we also require the non-small-frequency contribution. In 41, the self-force was calculated in the particular case of a static particle in (a static patch of) the Nariai spacetime. In such spacetime the 'retarded' Green function possesses no BC (since the radial potential falls off exponentially near the 'cosmological horizons'); however, in the Schwarzschild spacetime the Green function does possess a BC, the knowledge of which is required if we wish to perform a similar calculation of the self-force to the one performed in 41] in Narai. In this paper we have derived the large-frequency asymptotics for the BC contribution to the Green function modes for spins 0, 1 and 2 in the Schwarzschild spacetime - see Eq. 3.39) for the leading order. These asymptotics lead to a divergence of the BC Green function at the 'very early' times $\Delta t<\left|r_{*}\right|+\left|r_{*}^{\prime}\right|$ and they lead to a convergence of the $\nu$-integral in the upper limit when $\Delta t>\left|r_{*}\right|+\left|r_{*}^{\prime}\right|$. In Sec. $\mathrm{V}$ we have investigated the contributions of the high-frequency BC and the high-frequency QNM to the perturbation response given an initial perturbation. We have shown that if the initial data is of compact support within $r_{*}<R_{*}$, then both contributions separately converge when $\Delta t>\left|r_{*}\right|+R_{*}$; however, when the initial data is a non-compact Gaussian distribution, both contributions diverge separately for any fixed time $\Delta t$ and radius $r_{*}$. We expect that the divergences from all contributions to the Green function (i.e., from the QNM series, the BC and the high-frequency arc) to cancel each other out so that the full perturbation response is finite - we leave this study for future work.

We have also obtained in Eq. (4.4) the highly-damped QNM frequencies for spin-1 (and reproduced existing results for the corresponding spin-0 and spin-2 frequencies) in Schwarzschild for the first time in the literature (other than the previously-known leading-order for the imaginary part). In order to obtain the leading order of the spin-1 asymptotics we had to go up to two orders higher for large- $\bar{\nu}$ than is necessary for $s=0$ and 2 . The real part of these spin- 1 frequencies approaches the NIA, unlike for $s=0$ and 2 , and it does so faster (like $n^{-3 / 2}$ ) than for $s=1 / 2$ and $5 / 2$ (which go like $n^{-1 / 2}$ ).

\section{Acknowledgments}

We thank Emanuele Berti and Vítor Cardoso for making available the numerical data in 39. M.C. acknowledges funding support from the Irish Research Council for Science, Engineering and Technology, co-funded by Marie Curie Actions under FP7. A.O. acknowledges support from Science Foundation Ireland under grant no 10/RFP/PHY2847.

\section{Appendix A: Check on the relationship between $A_{\ell, \omega}^{\text {out }}$ and $A_{\ell, \omega}^{\text {in }}$}

In this appendix we calculate the relationship between the coefficients $A_{\ell, \omega}^{\text {out }}$ and $A_{\ell, \omega}^{\text {in }}$ by following closely the method in [12]: we will analytically continue the radial function $f_{\ell}$ starting at point $\mathrm{A}$ in Fig, 1 , then down to point $\mathrm{B}$, where $\arg (r)=3 \pi / 4$, then around an argument of ' $-3 \pi / 2$ ' to reach the anti-Stokes line at $\arg (r)=-3 \pi / 4$, then down that anti-Stokes line to radial infinity (i.e., to the 'reflection point' of point A), and finally all around radial infinity anticlockwise back to point A (this is Fig.1 [12]). We will then impose the exact monodromy Eq. (3.29). This is a check on the relationship between $A_{\ell, \omega}^{\text {out }}$ and $A_{\ell, \omega}^{i n}$ found in Eqs. 3.36 and 5.10 following a different contour. We will do it here only for $s=2$.

We start with the boundary condition $f_{\ell}(r,-i \nu) \sim A_{\ell, \omega}^{o u t} g_{a}(r,-i \nu)+A_{\ell, \omega}^{i n} g_{a}(r,+i \nu)$ at the point A in Fig 1 from Eq. 2.4. At $\arg (r)=3 \pi / 4$ we have

$$
\begin{aligned}
& \frac{\sqrt{\pi \bar{\nu}^{3}}}{4} \psi_{1}^{(0)}(t) \sim-e^{t^{2} / 2}-i e^{-t^{2} / 2} \sim-g_{a}(r, i \nu) e^{i \pi \bar{\nu}}-i g_{a}(r,-i \nu) e^{-i \pi \bar{\nu}} \\
& \frac{4}{\sqrt{\pi \bar{\nu}}} \psi_{2}^{(0)}(t) \sim-3 i e^{t^{2} / 2}+e^{-t^{2} / 2} \sim-3 i g_{a}(r, i \nu) e^{i \pi \bar{\nu}}+g_{a}(r,-i \nu) e^{-i \pi \bar{\nu}}
\end{aligned}
$$

The first step follows from Eqs. 3.17) and (3.21); in the second step we have used Eq.(3.25). We solve for $g_{a}(r, \pm i \nu)$ 
and replace in the above boundary condition to obtain

$$
f_{\ell}(r,-i \nu) \sim \psi_{1}^{(0)}(t) \frac{\sqrt{\pi \bar{\nu}^{3}}}{8}\left[3 i A_{\ell, \omega}^{\text {out }} e^{i \pi \bar{\nu}}+A_{\ell, \omega}^{i n} e^{-i \pi \bar{\nu}}\right]+\psi_{2}^{(0)}(t) \frac{2}{\sqrt{\pi \bar{\nu}}}\left[-A_{\ell, \omega}^{\text {out }} e^{i \pi \bar{\nu}}+i A_{\ell, \omega}^{i n} e^{-i \pi \bar{\nu}}\right]
$$

We have obtained this expression for $\arg (r)=3 \pi / 4$, but it is valid $\forall \arg (r)$ by analytic continuation. We now explicitly evaluate it at $\arg (r)=-3 \pi / 4$. From Eqs.3.14 together with Eq. A1] we obtain

$\psi_{1}^{(0)}\left(t e^{-3 \pi i / 2}\right)=-i \psi_{1}^{(0)}(t), \quad \frac{\sqrt{\pi \bar{\nu}^{3}}}{4} \psi_{1}^{(0)}(t) \sim-e^{t^{2} / 2}+i e^{-t^{2} / 2} \sim-g_{a}(r, i \nu) e^{-i \pi \bar{\nu}}+i g_{a}(r,-i \nu) e^{i \pi \bar{\nu}}$

$\psi_{2}^{(0)}\left(t e^{-3 \pi i / 2}\right)=-i \psi_{2}^{(0)}(t)-\frac{3 \bar{\nu}^{2} \pi}{8} \psi_{1}^{(0)}(t), \quad \frac{4}{\sqrt{\pi \bar{\nu}}} \psi_{2}^{(0)}(t) \sim 5 i e^{t^{2} / 2}+3 e^{-t^{2} / 2} \sim 5 i g_{a}(r, i \nu) e^{-i \pi \bar{\nu}}+3 g_{a}(r,-i \nu) e^{i \pi \bar{\nu}}$

at $\arg (r)=-3 \pi / 4$, where in the last step we have again used the top equation in (3.25). Introducing these expressions into Eq. A2 we obtain

$$
f_{\ell}(r,-i \nu) \sim g_{a}(r,-i \nu) e^{-i \pi \bar{\nu}}\left[-3 A_{\ell, \omega}^{\text {out }} e^{i \pi \bar{\nu}}+2 i A_{\ell, \omega}^{i n} e^{-i \pi \bar{\nu}}\right]+g_{a}(r, i \nu) e^{i \pi \bar{\nu}}\left[i A_{\ell, \omega}^{\text {out }} e^{i \pi \bar{\nu}}-3 A_{\ell, \omega}^{i n} e^{-i \pi \bar{\nu}}\right]
$$

at the 'reflection point' of point $\mathrm{A}$ in Fig. 1. We can analytically continue this expression all around infinity anticlockwise and back to point $\mathrm{A}$. We then equate this expression to the original boundary condition given above after applying the monodromy Eq. 3.29 , i.e., we equate Eq. A4 to $f_{\ell}\left(\left(r-r_{h}\right) e^{2 \pi i},-i \nu\right) \sim e^{-2 \pi i \bar{\nu}} A_{\ell, \omega}^{\text {out }} g_{a}(r,-i \nu)+$ $e^{-2 \pi i \bar{\nu}} A_{\ell, \omega}^{i n} g_{a}(r,+i \nu)$. Equating the coefficient of $g_{a}(r,-i \nu)$ gives precisely the relationship between $A_{\ell, \omega}^{\text {in }}$ and $A_{\ell, \omega}^{\text {out }}$ that follows from Eqs. (3.36) and (5.10). Note that the coefficient of $g_{a}(r, i \nu)$ is not to be trusted since when closing the contour at infinity it is $\operatorname{Re}\left(r_{*}\right)>0$ and so $g_{a}(r, i \nu)$ is the subdominant solution there.

[1] E. Poisson, A. Pound, and I. Vega (2011), 1102.0529.

[2] E. W. Leaver, Phys. Rev. D 34, 384 (1986).

[3] C. V. Vishveshwara, Nature 227, 936 (1970).

[4] R. H. Price, Phys. Rev. D5, 2419 (1972).

[5] R. H. Price, Phys. Rev. D5, 2439 (1972).

[6] N. Andersson, Phys. Rev. D 55, 468 (1997).

[7] P. T. Leung, A. Maassen van den Brink, K. W. Mak, and K. Young (2003), gr-qc/0307024.

[8] P. T. Leung, A. Maassen van den Brink, K. W. Mak, and K. Young, Class. Quant. Grav. 20, L217 (2003), gr-qc/0301018.

[9] A. Maassen van den Brink, Phys. Rev. D62, 064009 (2000), gr-qc/0001032.

[10] A. Maassen van den Brink, J. Math. Phys. 45, 327 (2004), gr-qc/0303095.

[11] L. Motl and A. Neitzke, Ad. Theor. Math. Phys. 7, 307 (2003).

[12] A. Neitzke (2003), hep-th/0304080.

[13] S. Hod, Phys. Rev. Lett. 81, 4293 (1998), gr-qc/9812002.

[14] O. Dreyer, Physical Review Letters 90, 081301 (2003), arXiv:gr-qc/0211076.

[15] M. Maggiore, Phys. Rev. Lett. 100, 141301 (2008), 0711.3145.

[16] J. D. Bekenstein, Lett. Nuovo Cim. 11, 467 (1974).

[17] J. D. Bekenstein and V. F. Mukhanov, Phys. Lett. B360, 7 (1995), gr-qc/9505012.

[18] T. Padmanabhan, Class. Quant. Grav. 21, L1 (2004), gr-qc/0310027.

[19] U. Keshet and A. Neitzke, Phys. Rev. D78, 044006 (2008), 0709.1532.

[20] J. Babb, R. Daghigh, and G. Kunstatter, Phys. Rev. D84, 084031 (2011), 1106.4357.

[21] S. Musiri and G. Siopsis, Class. Quant. Grav. 20, L285 (2003), hep-th/0308168.

[22] L. Motl, Adv. Theor. Math. Phys. 6, 1135 (2002), gr-qc/0212096.

[23] S. Musiri and G. Siopsis, Phys. Lett. B650, 279 (2007).

[24] V. Cardoso, J. P. S. Lemos, and S. Yoshida, Phys. Rev. D69, 044004 (2004), gr-qc/0309112.

[25] E. Berti, V. Cardoso, and A. O. Starinets, Class. Quant. Grav. 26, 163001 (2009), 0905.2975.

[26] R. A. Konoplya and A. Zhidenko, Rev. Mod. Phys. 83, 793 (2011), 1102.4014.

[27] J. D. Schnittman, Class. Quant. Grav. 28, 094021 (2011), 1010.3250.

[28] M. Casals and A. C. Ottewill, in preparation.

[29] M. Casals and A. C. Ottewill, in preparation.

[30] T. Regge and J. A. Wheeler, Phys. Rev. 108, 1063 (1957).

[31] J. A. Wheeler, Phys. Rev. 97, 511 (1955).

[32] N. Andersson and C. J. Howls, Class. Quant. Grav. 21, 1623 (2004), gr-qc/0307020. 
[33] N. Andersson, M. E. Araujo, and B. F. Schutz, Classical and Quantum Gravity 10, 735 (1993).

[34] E. W. Leaver, J. Math. Phys. 27, 1238 (1986).

[35] E. S. C. Ching, P. T. Leung, W. M. Suen, and K. Young, Phys. Rev. Lett. 74, 2414 (1995), gr-qc/9410044.

[36] E. S. C. Ching, P. T. Leung, W. M. Suen, and K. Young, Phys. Rev. D52, 2118 (1995), gr-qc/9507035.

[37] http://dlmf.nist.gov/

[38] E. S. C. Ching, P. T. Leung, W. M. Suen, and K. Young, Phys. Rev. D 54, 3778 (1996).

[39] http://www.phy.olemiss.edu/?berti/qnms.html, http://gamow.ist.utl.pt/?vitor/ringdown.html

[40] E. Berti and V. Cardoso, Phys. Rev. D 74, 104020 (2006).

[41] M. Casals, S. Dolan, A. C. Ottewill, and B. Wardell, Phys. Rev. D79, 124043 (2009), 0903.0395. 